\title{
Hopfield Neural Network Method for Problem of Telescoping Path Optimization of Single-Cylinder Pin-Type Multisection Boom
}

\author{
Yan Mao iD and Kai Cheng (iD) \\ School of Mechanical and Aerospace Engineering, Jilin University, Changchun 130025, China \\ Correspondence should be addressed to Kai Cheng; chengkai@jlu.edu.cn
}

Received 6 April 2019; Accepted 13 June 2019; Published 8 July 2019

Academic Editor: Alessandro Formisano

Copyright (C) 2019 Yan Mao and Kai Cheng. This is an open access article distributed under the Creative Commons Attribution License, which permits unrestricted use, distribution, and reproduction in any medium, provided the original work is properly cited.

\begin{abstract}
Telescoping path optimization (TPO) of single-cylinder pin-type multisection boom (SPMB) is a practical engineering problem that is valuable to investigate. This article studies the TPO problem and finds the key of TPO is to obtain the maximum retraction backmost combination. A mathematic model on the basis of the quadratic penalty function of a Hopfield neural network (HNN) is constructed. Two strategies are presented to improve the performance of TPO model: one is proportional integral derivative (PID) strategy that adaptively adjusts the parameter $\lambda$ of the constrained term and the parameter $\gamma$ of the optimization objective term by controlling the value of constraint violation $g_{k}$ and the other is efficiency factor strategy that an efficiency factor is introduced in model for prioritizing the constrained term over the objective term. Data test shows that compared with the path of boom length changing before optimization, both the number of sections that need to be moved and the total travels of cylinder can be reduced by 10\%-30\% after optimization. Both the PID strategy and the efficiency factor strategy achieve good optimization effects. The efficiency factor strategy is excellent at moderating the conflicts between the constrained term and the objective term; thus the generations of the valid and the optimal solutions get well improved.
\end{abstract}

\section{Introduction}

The upstructure of a mobile crane is composed of four major mechanisms: slewer, derricking mechanism, winch, and telescopic boom. Single-cylinder pin-type telescopic multisection boom (SPMB) is the structure of telescopic boom. The SPMB has a fixed section and multiple telescopic sections, and the telescopic sections are sleeved one by one in the way that the small section is inserted in the large one. The length of each section is divided into several scales, and each scale has a hole set for being locked by pin of the inner adjacent section. Therefore, the different hole of outer section being locked by the pin of inner section determines the different stretching of the inner section. A single long cylinder driving boom sections sequentially and the sections are pined gradually to keep the extension of the boom. Boom length is determined by all sections' stretching, and each section's stretching is decided by the location of the hole being pinned of its outer section. Obviously the boom length values of SPMB are discontinuous.

Single-cylinder pin-type multisection boom (SPMB) has good load bearing performance that is mainly used in large tons automobile crane at present. For example, the truck cranes with over 100 tons lift weight are most equipped with SPMB mechanisms; a giant all terrain crane with lift weight of 2,000 tons has an eight-section SPMB, whose boom length exceeds 100 meters when the SPMB is being fully stretched. For now, the material of SPMB is steel for crane, and the maximum number of boom sections is only eight owing to weight limit of material. In future, with the design optimization and the applications of light-weight materials, such as carbon fiber and polymer materials, the sections number might be manufactured more than the eight, might be, $n=10,20,30$ and so on.

However, the telescoping efficiency of SPMB is low. Because all the sections are driven by a long single cylinder, 
the multiple sections must await to be retracted one by one in sequence from initial position to backmost position and then wait to be stretched in reverse sequence from the backmost position to target position, while changing boom length from one state to another. When telescoping one section, a set of complex procedure (telescoping step) must be operated, mainly involving processes of cylinder freely moving towards aimed section and cylinder driving the aimed section towards target position. Provided that a boom has $n$ sections, $(2 n-$ 1) telescoping steps should be operated when changing the boom length, where $(n-1)$ steps are for retracting sections and $n$ steps are for stretching sections. If optimization is performed, the telescoping steps can be reduced by one or two, and then the work efficiency can be evidently improved.

TPO of SPMB is a practical engineering problem. Although SPMB is widely applied in machinery and equipment, research on TPO is lacking.

Reference [1] (2012) described the TPO problem first in public and optimized a five-section SPMB in a nested program. The principle was that, judging the sections from the outermost section to the innermost section, if the required cylinder travel exceeded the offered cylinder travel, retracted the section fully; otherwise, it kept the section unmoved [1]. Reference [1] is enumeration method and makes judgment only between two states of "retract fully" and "keep unmoved." Thus the effect of optimization is very limited.

Mao Y et al. (2018) proposed a simplified Permutation and Combination (P\&C) method that chose three states for each section to participate P\&C; those were "hold at initial position," "move to target position," and "retract to full back" (actually, there are usually more states than these three. If there are number $m$ holes on a section, there will be number $m$ stretched states for the section). Took these three states of all sections to form state combinations and to participate $\mathrm{P} \& \mathrm{C}$ together, then eliminate the state combinations exceeding cylinder travel allowance (invalid solutions) by function evaluations, and finally, pick out the state combinations having the shortest paths (optimal solutions) from the remaining state combinations (valid solutions) [2]. P\&C algorithm belongs to enumeration category, which can get optimal solutions in short time when boom sections are few. But if the number of boom sections increases, its calculation will grow up exponentially. Besides, the simplified handling that only three states for each section are chosen to form state combinations of all sections cannot describe all telescoping path possibilities.

Reference [3] (2018) is another method to calculate the TPO. The method evaluated permitted cylinder travel allowance starting from the endmost section back to the foremost section. Through two function evaluations of maximum permitted travel allowance and minimum permitted travel allowance, it was decided whether opening the side function evaluations or retracting one section full back and then continuing downward evaluations [3].

References [1-3] are enumeration methods in nature and are suitable for small-scale problems because their logic will be increasingly complicated with problem scales enlarging. Besides, these methods only pick out limited number of states of the holes locations to make judgments in order to simplify calculations and cannot reflect all the path possibilities, so their optimization effects are not very well.

TPO refers to the fact that when telescoping a multisection boom from an initial state to a target state, the number of telescoping steps is the smallest and the total travels of the driving cylinder is the shortest. TPO is essentially the shortest-path scheduling problem of combination optimization problem (COP). TPO aims to work out maximum retraction backmost combination ( $\mathrm{RBC}$ ); that is, the $\mathrm{RBC}$ position always exists while all sections retract from the initial position combination. After retracting sections to the RBC position, sections should be stretched to target position combination subsequently. RBC length is limited in the full travel of the long cylinder that is set as "1." The TPO problem has the following features. Movements of sections should be operated in sequence. When one section is ready to be retracted or stretched, the current sufficiency of the travel allowance of the driving cylinder should be considered. Moreover, one section's movement can affect other sections' movability. Thus, TPO contains multiple constraints that associate with each other.

Small-scale COPs can be solved by exact algorithms, such as dynamic programming, branch definition, and enumeration. Heuristics can quickly calculate approximate optimal solutions for large-scale complex problems, but their optimality cannot be guaranteed. Heuristics include neighbor algorithms, simulated annealing, evolutionary algorithms, and neural networks.

The Hopfield neural network (HNN) has advantages in solving COPs. In practical applications, when converting the objective function of the optimization problem to the energy equation of HNN to map variables to neural states in the network, HNN can be used to solve COPs. That is, when the neural state of the network tends to equilibrium, the energy equation of the network converges to minimum; the network's convergence from initial state to steady state illustrates the optimization calculating process of the objective function [4].

The energy equation is convenient to constraints disposing, yet HNN has following difficulties: (I) the penalty parameters of energy equation are difficult to determine; (II) the convergence is often trapped in local minimum; (III) for problems of the optimal solutions distributing on boundary of constraints, the adjustments of constrained term sometimes conflict with the adjustment of objective term, which may cause oscillations near convergence point.

This study investigates the TPO problem. A mathematic model on the basis of the quadratic penalty function of $\mathrm{HNN}$ is constructed. Two strategies are presented to improve the performance of TPO model; one is the proportional integral derivative (PID) strategy that adaptively adjusts the parameter $\lambda$ of the constrained term and parameter $\gamma$ of the optimization objective term by controlling the value of constraint violation $g_{k}$; the other is efficiency factor strategy that an efficiency factor is introduced in model for prioritizing the constrained terms over the objective term.

Data test shows that compared with the path of boom length changing before optimization, both the number of 


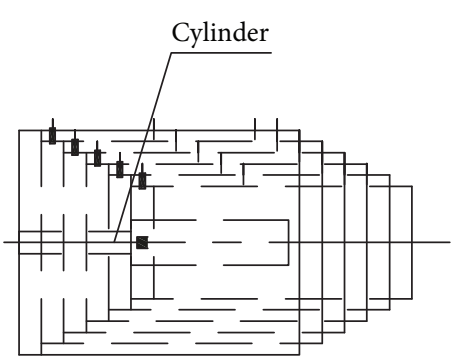

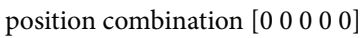
state combination [ $\left[\begin{array}{lllll}1 & 1 & 1 & 1 & 1\end{array}\right]$

(a) Full retraction

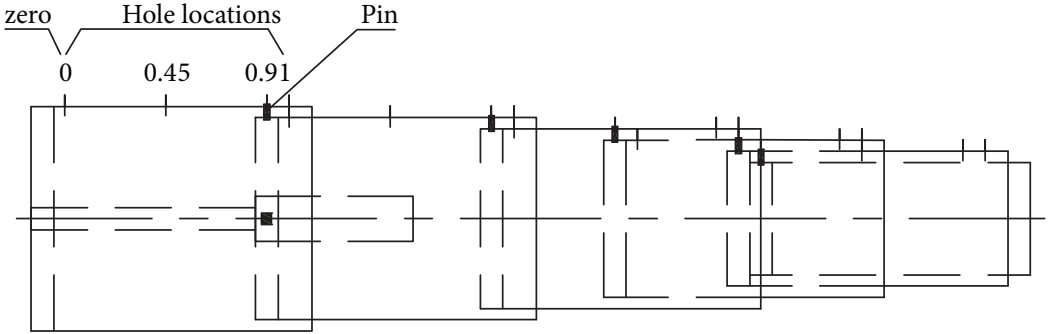

position combination [0.9 0.90 .450 .450 ] state combination [ $\left[\begin{array}{lllll}3 & 3 & 2 & 2 & 1\end{array}\right]$

(b) A boom stretching state

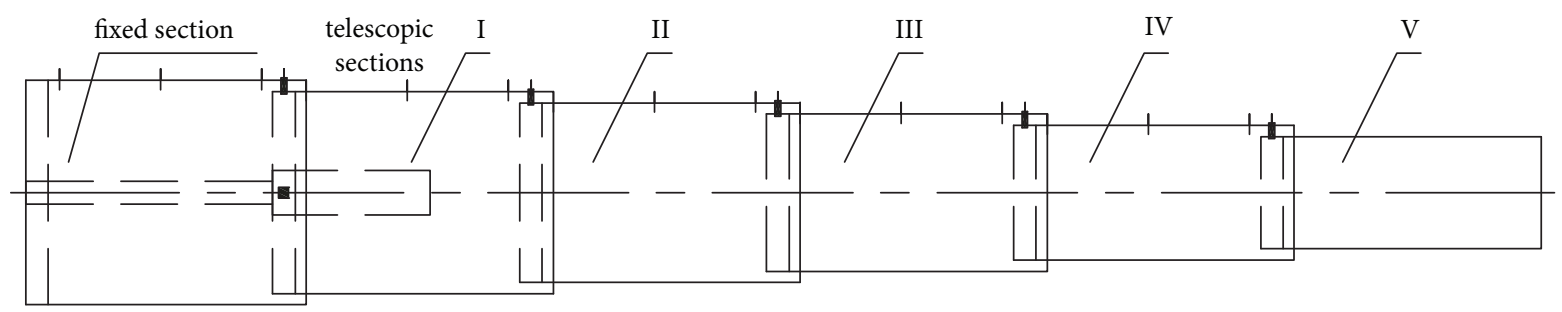

position combination [ $\left.\begin{array}{lllll}1 & 1 & 1 & 1 & 1\end{array}\right]$

state combination [4 4444 ]

(c) Full extension

FIgURE 1: Structure of a SPMB with five telescopic sections.

sections that need to be moved and the total travels of cylinder can be reduced by $10 \%-30 \%$ after optimization. Both the PID strategy and the efficiency factor strategy achieve good optimization effects. It is found that the main reason leading to the generations of low quality solution is the oscillations being triggered during convergence process. The efficiency factor strategy is excellent at moderating the conflict between the constrained term and the objective term and restrains the oscillations successfully thereby. The study consists of the following five parts.

Section 1: Introduction.

Section 2: SPMB mechanism and TPO problem.

Section 3:: HNN method applied for TPO problem.

Section 4: Simulation, analysis, and discussion.

Section 5: Conclusion and prospect,

\section{SPMB Mechanism and TPO Problem}

2.1. SPMB Mechanism. Figure 1 illustrates an example of SPMB with five telescopic sections, which are denoted separately in signs of I, II, III, IV, and V. The length of each section is divided into four scales, and each scale has a hole set for being locked by pin of the inner adjacent section. Therefore, the different hole of the outer section being locked by the pin of the inner section determines the different stretching of the inner section. Let one-section length be defined as 1 , and the hole locations are $0,0.45,0.9$, and 1 . The five telescopic sections of the boom length may be expressed as a combination $\{0.9,0.9,0.45,0.45,0\}$, which means that Sections I and II are extended by 0.9 , Sections III and IV are extended by 0.45 , and Section $V$ is not extended. To simplify the expression of section length combination, the holes locations $0,0.45,0.9$, and 1 are replaced by four states of " 1 ," " 2 ," " 3 ," and " 4 ," respectively. Then, the mentioned section length combination is expressed in an array $\left[\begin{array}{llll}3 & 3 & 2 & 2\end{array}\right.$ $1]$.

The array [ $\left.\begin{array}{llllll}1 & 1 & 1 & 1 & 1 & 1\end{array}\right]$ in Figure 1(a) denotes the boom's full

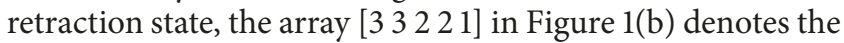
boom's stretched state, and the array [ $\begin{array}{llll}4 & 4 & 4 & 4\end{array} 4$ ] in Figure 1(c) denotes the boom in full extension.

2.2. TPO Problem. A telescoping step means the whole procedure being executed when a section is moved. The procedure involves processes of the cylinder freely moving towards aimed section and the cylinder driving the aimed section to target position. The procedure of the cylinder movement is similar to a round trip; for example, when stretching a section, the leaving trip is the cylinder driving the section forwards to target position $\left(S_{\text {boom }}\right)$, whereas the returning trip is the cylinder retracting itself backwards to the position of next section $\left(S_{\text {cylinder }}\right)$. When retracting a section, the leaving trip is the cylinder driving the section backwards to target position $\left(S_{\text {boom }}\right)$ whereas the returning trip is the cylinder stretching itself to the position of next section $\left(S_{\text {cylinder }}\right)$. Therefore, the optimization objective of TPO $S_{\text {total }}$ includes two parts: the shortest cylinder telescoping path $S_{\text {cylinder }}$ and the shortest boom section telescoping path $S_{\text {boom }}$. 
TABLE 1: Effect comparison of the optimized paths with the non-optimized path.

\begin{tabular}{|c|c|c|c|c|c|c|c|c|}
\hline $\begin{array}{l}\text { Step } \\
\text { NO. }\end{array}$ & $\begin{array}{c}\text { Non- } \\
\text { optimized }\end{array}$ & $\begin{array}{l}\text { Driven } \\
\text { section }\end{array}$ & $\begin{array}{c}\text { Optimization } \\
1\end{array}$ & $\begin{array}{l}\text { Driven } \\
\text { section }\end{array}$ & $\begin{array}{c}\text { Optimization } \\
2\end{array}$ & $\begin{array}{l}\text { Driven } \\
\text { section }\end{array}$ & $\begin{array}{c}\text { Optimization } \\
3\end{array}$ & $\begin{array}{l}\text { Driven } \\
\text { section }\end{array}$ \\
\hline 0 & 22222 & -- & 22222 & -- & 22222 & -- & 22222 & -- \\
\hline 1 & 12222 & I & 12222 & I & 12222 & I & 21222 & II \\
\hline 2 & 11222 & II & 11222 & II & 11222 & II & 21122 & III \\
\hline 3 & 11122 & III & 11122 & III & 11212 & IV & 21112 & IV \\
\hline 4 & 11112 & IV & 11112 & IV & 21212 & I & 21212 & III \\
\hline 5 & 11111 & $\mathrm{~V}$ & 11212 & III & & & & \\
\hline 6 & 11112 & $\mathrm{~V}$ & 21212 & I & & & & \\
\hline 7 & 11212 & III & & & & & & \\
\hline 8 & 21212 & I & & & & & & \\
\hline Path boom & 3.6 & & 2.7 & & 1.8 & & 1.8 & \\
\hline Path cylinder & 3.6 & & 3.6 & & 2.7 & & 2.7 & \\
\hline Total path & 7.2 & & 6.3 & & 4.5 & & 4.5 & \\
\hline
\end{tabular}

Note: Sign "italic" denotes RBC.

A boom with $n$-telescopic sections must run $(2 n-1)$ times the above procedures (telescoping steps), $n$ times are for retracting $n$-sections full back; and $(n-1)$ times are for stretching $n$-sections to target positions (because the endmost section can save one step for direct stretching from initial state to target state), if without optimization. Then, a boom with 5-telescopic sections must run nine telescoping steps, if without optimization. The repeated operations of procedures mean heavy work and considerable time consumption. If TPO is performed, the telescoping steps can be reduced by one or two. Thus, energy consumption and labor intensity can be reduced, and work efficiency can be promoted in engineering applications. Table 1 shows an effect comparison before and after TPO.

In Table 1 , the initial state combination is $\left[\begin{array}{lllll}2 & 2 & 2 & 2 & 2\end{array}\right]$, and the target state combination is $\left[\begin{array}{lllll}2 & 1 & 2 & 1 & 2\end{array}\right]$. Without any optimization, $\mathrm{RBC}=\left[\begin{array}{lllll}1 & 1 & 1 & 1 & 1\end{array}\right]$. Eight steps are required to change from initial boom length to target boom length. The boom path length $\left(S_{\text {boom }}\right)$ is 3.6, and the cylinder path length $\left(S_{\text {cylinder }}\right)$ is 3.6. Thus, the total path length $\left(S_{\text {total }}\right)$ is 7.2.

With optimization $1, \mathrm{RBC}=\left[\begin{array}{lllll}1 & 1 & 1 & 1 & 2\end{array}\right]$, six steps are necessary, $S_{\text {boom }}$ is 2.7 , and the $S_{\text {cylinder }}$ is 3.6. Therefore, $S_{\text {total }}$ is 6.3 .

With optimization $2, \mathrm{RBC}=\left[\begin{array}{lllll}1 & 1 & 2 & 1 & 2\end{array}\right]$ and with optimization 3, RBC = [2 1112]. Both RBCs only require four steps, $S_{\text {boom }}$ is 1.8 , and $\mathrm{S}_{\text {cylinder }}$ is 2.7 . Thus, $\mathrm{S}_{\text {total }}$ is 4.5 .

Note that optimizations 2 and 3 are the optimal solutions, the steps required are the least, and the total paths are the shortest.

2.3. $R B C$. $\mathrm{RBC}$ is an extreme combination of retraction positions for sections when changing the boom length. After all sections are retracted to RBC state, sections should extend subsequently. The full retraction state [11111] is always a valid $\mathrm{RBC}$ for sections, though the state is not an optimal RBC. Clearly, the larger the RBC, the shorter the path is, because only few retractions of sections being operated. Therefore, the optimization goal is to find the maximum $\mathrm{RBC}$ so as to achieve the minimum distances of boom retracting from the initial position to the RBC position and boom stretching from the RBC position to the target position. When the RBC is derived, the telescoping path can be listed out subsequently, as Table 1 listing.

2.4. Single-Cylinder Travel Constraints during the Telescoping Process (Constraints). Cylinder travel length must satisfy each step in each section during the telescoping process. Retraction or extension of any section is also performed. Whether the length of its former section is within the travel of the cylinder must be considered. The build-up evaluation equation of the cylinder travel allowance for each telescoping step is as follows:

$$
g_{k}=\left(\mathrm{C}_{j}[1]+C_{j}[2]+\ldots+M_{j}[k]-1\right) \leq 0
$$

In (1), $j$ is the current telescopic step, $k$ is the current driven section, $C_{j}[1], \ldots, C_{j}[k-1]$ is the sum of the extension length of former $(k-1)$ sections, and $M_{j}[k]$ $=\max \left(C^{T}{ }_{j}[k]\right.$, and $\left.C^{A}{ }_{j}[k]\right)$ is the maximum extension required in Section $k$. Herein, $C^{T}{ }_{j}[k]$ is the target section length, and $C^{A}{ }_{j}[k]$ is the initial section length.

2.5. Telescoping Path Definition (Optimization Objective). Figure 2 demonstrates the telescoping process of a 5-section boom and the total paths cylinder going through. Blue cycles indicate the initial and target positions of the sections. Orange cycles indicate the RBC positions of the sections. The white ring means the starting point of a cylinder (defined in zero). Values in brackets represent the potential energy height of the cylinder (absolute length). This measure cannot be over 1 , which is the cylinder travel limit, at any time. Solid lines represent cylinder path $S_{\text {cylinder }}$, and dotted lines show boom path $S_{\text {boom }}$. Their equations are provided below:

$$
S_{\text {total }}=S_{\text {boom }}+S_{\text {cylinder }}
$$




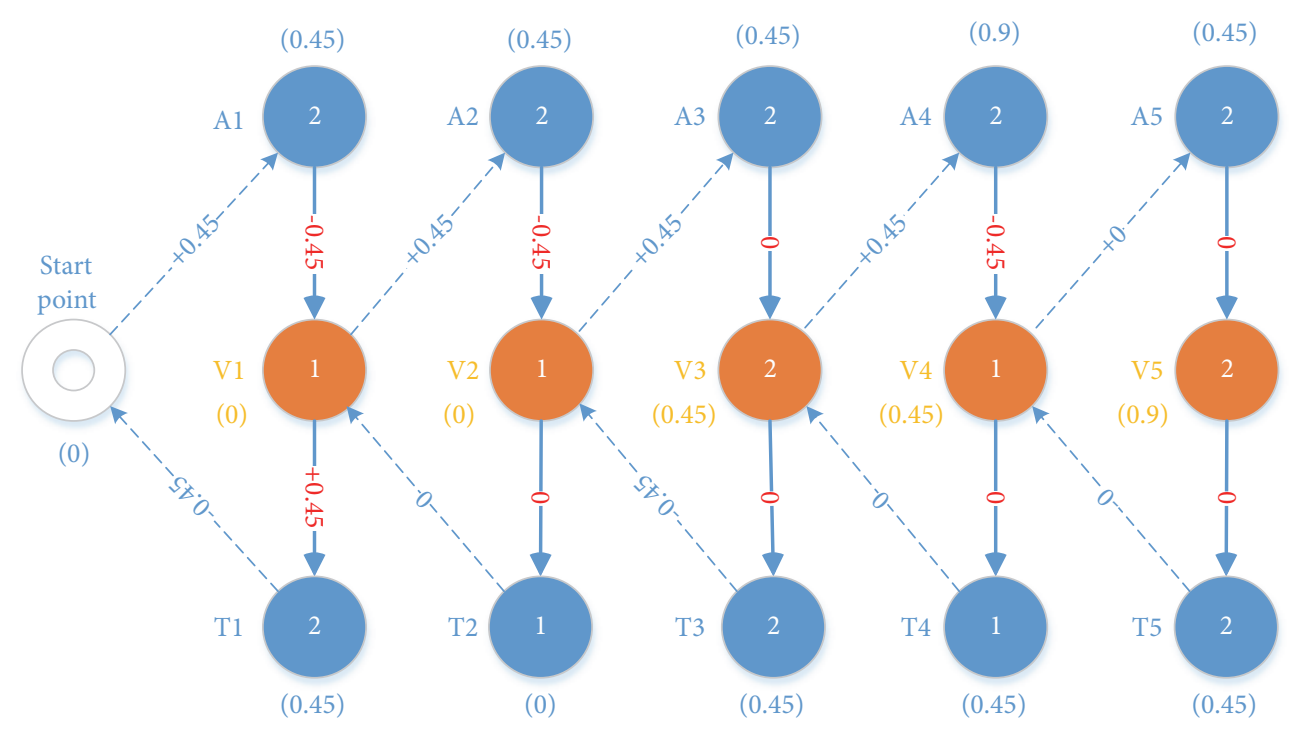

FIGURE 2: Paths of SPMB telescoping process.

$$
\begin{gathered}
S_{\text {boom }}=\sum_{j=1}^{5}\left(\left|A_{j} d-V_{j} d\right|+\left|T_{j} d-V_{j} d\right|\right) \\
S_{\text {cylinder }}=\sum_{j=1}^{5}\left(\left|A_{j} d-V_{o j} d\right|+\left|T_{j} d-V_{o j} d\right|\right)
\end{gathered}
$$

$A_{j}$ is the initial section state, $T_{j}$ is the target section state, $V_{j}$ is the RBC state, and $d$ is the hole location combination. $V=$ $\left[\begin{array}{lllll}V_{1} & V_{2} & V_{3} & V_{4} & V_{5}\end{array}\right], V_{\mathrm{o}}=\left[\begin{array}{lllll}V_{1} & V_{2} & V_{3} & V_{4} & 0\end{array}\right]$. Figure 2 describes that the boom sections retract from $A=\left[\begin{array}{llll}2 & 2 & 2 & 2\end{array}\right]$ to $V=\left[\begin{array}{lll}1 & 1\end{array}\right.$

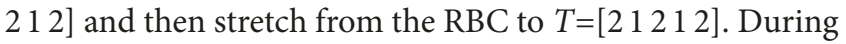
the telescoping process, the $S_{\text {boom }}=1.8$, the $S_{\text {cylinder }}=2.7$, and the total path is 4.5 .

\section{HNN Method Applied for TPO Problem}

3.1. HNN Method. In 1982, American physicist John Joseph Hopfield proposed a neural network model, which vigorously promoted the study of neural networks [5]. Then Hopfield and Tank (1985) successfully used this model to find the solutions of the traveling salesman problem (TSP) [6]. However, matching parameters is always difficult, and improper parameters set leads to bad performance of HNN. In order to properly determine the weight coefficients of energy function, scientists have made a series of research. Shirazi B. and H. S. (1989) used a matrix method to analyze the dynamics of continuous HNN and thus analyze the basic characteristics, advantages, and disadvantages of the model [7]. Aiyer et al. (1990) explained why the Hopfield network often falls into an invalid solution in the TSP problem by means of analyzing matrix eigenvalues and hypercube mapping. Then, they modified the energy function and derived parameters setting principles to ensure that the network converges to a valid solution [8]. Abe S. (1993) analyzed the hypercube vertex condition as it becomes a local minimum. The author also provided a method for suppressing the inferior solution on the basis of which weights and coefficients of the energy equation are set [9]. Sun S. et al. (1995) simplified the equation of Aiyer et al.s equation and demonstrated the validity of the penalty parameter determination based on Aiyer's matrix eigenvalue analysis method. Their equation almost achieved a $100 \%$ valid solution obtained from the 10-city TSP problem [10]. Subsequent researchers, such as Zhang J. et al. (1996), tested Sun's network, but it was unable to obtain $100 \%$ valid solutions an only calculated $70 \%$ valid solutions after extensive examination of a five-city TSP [11]. However, Sun's equation is still a simple and efficient form for TSP problems. Pedro M. Talavan and Javier Yanez (2002) introduced a parameter setting method for a stable condition analysis using the valid solution of an energy equation. Once the parameters are determined by the analytical method, any stable point becomes a valid path for TSP [12]. Effati and Baymain (2005) proposed the parameters chosen from the constrained differential equations [13]. Effati S. et al. (2007) demonstrated that the neural network in the form of quadratic penalty function is a Lyapunov function with uniform convergence [14].

Equation of Sun Shouyu for TSP is listed as follows [10]:

$$
\begin{aligned}
E= & \frac{A}{2} \sum_{x=1}^{n}\left(\sum_{i=1}^{m} V_{x i}-1\right)^{2}+\frac{B}{2} \sum_{i=1}^{m}\left(\sum_{x=1}^{n} V_{x i}-1\right)^{2} \\
& +\frac{D}{2} \sum_{x=1}^{n} \sum_{y=1}^{n} \sum_{i=1}^{m} V_{x i} d_{x y} V_{x i} V_{y, i+1}
\end{aligned}
$$

$A$ and $B$ are parameters of the constrained terms; $D$ is parameter of the objective term. Determinations of $A, B$, and $D$ follow principles of Aiyer's matrix eigenvalue analysis. Seeing that the principles of Aiyer are complicated, we apply the quadratic penalty function to construct model but take adaptive method to determine parameters. 
3.2. Permutation Matrix Representation of Section States. A permutation matrix is used to represent the sections and the corresponding positions of pins, where $V_{x i}$ is the pinned position $i$ of the Section $x$ of RBC $V ; x=1, \ldots, n$, with $n$ as the number of sections; $I=1, \ldots, m$, with $m$ as the number of pin holes; $A$ is the initial state matrix; $T$ is the target state matrix; $V$ is the RBC state matrix; and $d$ is the pinhole location array, such as $d=\left[\begin{array}{llll}0 & 0.45 & 0.9 & 1\end{array}\right]^{\prime}$ :

$$
\begin{aligned}
V & =\left(\begin{array}{cccc}
1 & 0 & 0 & 0 \\
1 & 0 & 0 & 0 \\
0 & 1 & 0 & 0 \\
1 & 0 & 0 & 0 \\
0 & 1 & 0 & 0
\end{array}\right) ; \\
V \times d & =\left(\begin{array}{c}
0 \\
0 \\
0.45 \\
0 \\
0.45
\end{array}\right)
\end{aligned}
$$

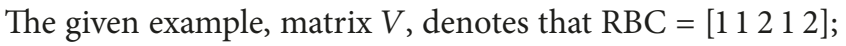
$V \times d$ denotes the extension length of boom sections.

3.3. Energy Equation of TPO. The energy function is given below:

$$
\begin{aligned}
& E_{\mathrm{TPO}}=\frac{B}{2} \sum_{x=1}^{n}\left(\sum_{i=1}^{m} V_{x i}-1\right)^{2}+\frac{\lambda}{2} \\
& \cdot \sum_{k=1}^{n}\left[\mathrm{~S}_{k}\left(\sum_{x=1}^{k} \sum_{i=1}^{m} V_{(x-1) i} d_{i}+M_{k}-1\right)\right]^{2}+\frac{c \gamma}{2} \\
& \cdot \sum_{x=1}^{n}\left[\left(\sum_{i=1}^{m} A_{x i} d_{i}-\sum_{i=1}^{m} V_{x i} d_{i}\right)^{2}\right. \\
&+\left(\sum_{i=1}^{m} T_{x i} d_{i}-\sum_{i=1}^{m} V_{x i} d_{i}\right)^{2} \\
&+\left(\sum_{i=1}^{m} A_{x i} d_{i}-\sum_{i=1}^{m} V_{x o i} d_{i}\right)^{2} \\
&\left.+\left(\sum_{i=1}^{m} T_{x i} d_{i}-\sum_{i=1}^{m} V_{x o i} d_{i}\right)^{2}\right] \\
& M_{k}=V_{k}=\left[\sum_{x=1}^{k} \sum_{i=1}^{m} V_{(x-1) i} d_{i}+M_{k}-1\right] \\
&\left.+\sum_{i=1}^{m} A_{k i} d_{i}, \sum_{i=1}^{m} T_{k i} d_{i}\right) \\
& \mathrm{k}=1 \ldots n ;
\end{aligned}
$$

$$
S_{k}= \begin{cases}0, & \mathrm{~g}_{k} \leq 0 \\ 1, & \mathrm{~g}_{k}>0 .\end{cases}
$$

$n$ is sections number and $m$ is holes number of each section, supposing the hole distributions on each section are the same. There are total three terms in (7).

(1) Term B. Equality constrained term is the row-constraint of permutation matrix $V$. The term defines that each row in matrix $V$ has one and only one element " 1 ," and the remaining elements are "0." Its physical meaning is that each section has one and only one hole being pinned.

(2) Term $\lambda$. Inequality constrained term is the rowconstrained term of $\operatorname{RBC}(V \times d)$, which denotes the outstretched length of the section. This term is composed of number $n$ inequality constraints that are associated with one another. Its physical meaning is defined as the constraints on each section and on the stretching of each step. During the retraction and extension of Section $x$, the sum of the extension length of all previous $(x-1)$ sections plus the maximum extension required length of current Section $x$ must be less than or equal to " 1 " (total travel of a single cylinder). From the second term in (7), we can see that

$k=1$ : when driving Section I, its maximum telescopic length should be less than the cylinder travel length " 1 ,"

$$
\begin{aligned}
& g_{1}=\left(M_{1}-1\right) \leq 0 \\
& S_{1} \equiv 0
\end{aligned}
$$

$k=2$ : when driving Section II, the current extension of Section I plus the maximum telescopic length of Section II should be less than the cylinder travel length "1,"

$$
g_{2}=\left(\sum_{x=1}^{2} \sum_{i=1}^{m} V_{(x-1) i} d_{i}+M_{2}-1\right) \leq 0
$$

$k=n$ : when driving Section $\mathrm{N}$, the current extensions of Sections I to (N-1) plus the maximum telescopic length of Section $\mathrm{N}$ should be less than the cylinder travel length " 1 ,"

$$
g_{n}=\left(\sum_{x=1}^{n} \sum_{i=1}^{m} V_{(x-1) i} d_{i}+M_{n}-1\right) \leq 0 .
$$

(3) Term $\gamma$. Optimization objective term is defined as the sum of squares of paths lengths $\left(S_{\text {cylinder }}\right.$ and $\left.S_{\text {boom }}\right)$ of each section retracting from the initial position to the $\mathrm{RBC}$ position and extending from the RBC position to the target position. Paths can only be minimized when the RBC of matrix $V$ takes a maximum.

$B, \lambda, \gamma$ are term parameters of (7), where $c$ is the efficiency factor. When $c=1$, efficiency factor does not work. Equation 
(11) reveals the relationship between the input $U_{i}$ and the output $V_{i}$ of neurons, in which the activation function is the hyperbolic tangent function. Furthermore, $\alpha$ is the ramp.

$$
V_{i}=f_{i}\left(U_{i}\right)=\frac{1}{2}\left(1+a \tanh \left(\frac{U_{i}}{T}\right)\right)
$$

3.4. Dynamic Differential Equation. Dynamic differential equation is derived as follows:

$$
\begin{aligned}
\Delta U & =\frac{d U_{x i}}{d t}=-\frac{\partial E}{\partial V_{x i}} \\
\Delta U & =-B\left(\sum_{i=1}^{m} V_{x i}-1\right) \\
& -\lambda \sum_{k=1}^{n}\left\{S_{k}\left(\sum_{x=1}^{k} \sum_{i=1}^{m} V_{(x-1) i} d_{i}+M_{k}-1\right) d_{i}{ }^{\prime}\right\} \\
& +2 \gamma c\left(\sum_{i=1}^{m} A_{x i} d_{i}\right. \\
& \left.+\sum_{i=1}^{m} T_{x i} d_{i}-\sum_{i=1}^{m} V_{x i} d_{i}-\sum_{i=1}^{m} V_{x o i} d_{i}\right) d_{i}^{\prime}
\end{aligned}
$$

Let $g_{k}=\left(\sum_{x=1}^{k} \sum_{i=1}^{m} V_{(x-1) i} d_{i}+M_{k}-1\right)$; the second term of (13) can be expanded as follows:

$$
\begin{aligned}
& \Delta U_{\lambda}=\left(\lambda_{1} S_{1} g_{1}+\lambda_{2} S_{2} g_{2}+\ldots+\lambda_{n} S_{n} g_{n}\right) d_{i}^{\prime} \\
& \quad=\left[\left(\lambda_{2} S_{2}+\lambda_{3} S_{3}+\ldots+\lambda_{n} S_{n}\right)\left(V_{1} \times d\right)\right. \\
& +\left(\lambda_{3} S_{3}+\lambda_{4} S_{4}+\ldots+\lambda_{n} S_{n}\right)\left(V_{2} \times d\right)+\ldots \\
& +\lambda_{n} S_{n}\left(V_{n-1} \times d\right)+\lambda_{1} S_{1}\left(M_{1}-1\right) \\
& \left.+\lambda_{2} S_{2}\left(M_{2}-1\right)+\ldots+\lambda_{n} S_{n}\left(M_{n}-1\right)\right] d_{i}^{\prime}
\end{aligned}
$$

Equation (14) indicates that when being at the RBC state, the restraint for the extension of Section I $\left(V_{1} \times d\right)$ is at its strongest, which is $\left(\lambda_{2} S_{2}+\lambda_{3} S_{3}+\ldots+\lambda_{n} S_{n}\right)$. The restraint for the extension of Section II is slightly weak at $\left(\lambda_{3} S_{3}\right.$ $\left.+\lambda_{4} S_{4}+\ldots+\lambda_{n} S_{n}\right)$. No restraint exists for the extension of Section N. The given explanation is consistent with the reality that the endmost section (Section $\mathrm{N}$ ) can arbitrarily retract and stretch since it will not influence the movement of other sections. Therefore, Section $\mathrm{N}$ is the most free. On the contrary, the length changing of the foremost section (Section I) will have a subsequent effect on the movements of all other sections. Thus, its freedom is the smallest, and its restraint is the strongest. Telescoping performed in sequence simply is to eliminate the associated influence on each section. In (14), Section I is subjected to the biggest penalty to minimize the stretching length $\left(V_{1} \times d\right)$ and release cylinder travel space for the retracting and stretching of subsequent sections.

Equation (15) is the dynamic updating equation, where $l_{r}$ is iteration step size.

$$
U(\mathrm{t}+1)=U(t)-l_{r} \times \Delta U
$$

3.5. PID Adaptive Parameter Adjustment Strategy. Matching penalty parameters is always difficult. The consequence of improper selection of parameters is that the convergence is trapped in local minimum and could not get optimal solution.

In order to escape the local optimal solution, there are generally some categories of strategies: one is to adjust the parameter and to change the weight values of the neural network. Since the weight values determine the shape of energy surface, the gradient descent path is changed thereby. PID adaptive parameter adjustment is in this way, which adaptively adjusts the descent path towards the lower point of energy loss by changing the shape of energy surface timely.

Other strategies are like that when the parameters and the input are constant; the shape of energy surface is determined. If the energy loss stops at a saddle point, there might have been a force to push it continuously descending, and the momentum strategy is in this way. If the energy loss is hard to converge to a balance point stably, the iteration step size can be regulated to help convergence, and the learning rate adjustment strategy is in this way [15].

Some other strategies are proposed by researchers to promote the searching effect of network. For example, the hill jumping algorithm divides energy function $E$ into two parts $-E_{1}$ and $E_{2}$. Energy functions $E, E_{1}$, and $E_{2}$ are alternatively run, and their weights and bias are recorded. The weight and bias of one energy function are set as the new starting point of another energy function. Finally, a global minimum point is promisingly reached [16]. In addition, there are noise gradient strategy [17], Stable Manifold Theorem [18], noise chaotic neural network [19], noise vector strategy [20], method of increase training times [21], and so on.

Principles of PID adaptive parameter adjustment are as follows:

Associate $\Delta \lambda$ with $g_{k}$ :

$$
\begin{aligned}
\Delta \lambda_{k}(t)= & K_{p \lambda} \cdot g_{k}(t)+K_{i \lambda} \cdot \sum_{t} g_{k}(t)+K_{d \lambda} \\
& \cdot\left(g_{k}(t)-g_{k}(t-1)\right) \\
\lambda(t+1)= & \lambda(t)+\mu_{\lambda} \Delta \lambda
\end{aligned}
$$

Associate $\Delta \gamma$ with $g_{k}$ :

$$
\begin{aligned}
\Delta \gamma(t)= & K_{p \gamma} \cdot g_{k}(t)+K_{i \gamma} \cdot \sum_{t} g_{k}(t)+K_{d \gamma} \\
& \cdot\left(g_{k}(t)-g_{k}(t-1)\right) \\
\gamma(t+1)= & \gamma(t)-\mu_{\gamma} \cdot \Delta \gamma(t) \cdot \gamma(t)
\end{aligned}
$$

$K_{p}$ is the proportional coefficient, $K_{i}$ is the integral coefficient, and $K_{d}$ is the differential coefficient. $\lambda$ is the parameter of the constrained term of (7), equations (16) and (17) are the setting laws of $\lambda$, and the PID adaptive adjustment of $\lambda$ is based on the control of constraint violation $g$ and is gradient-rising. $\gamma$ is the parameter of the objective term of (7), equations (18) and (19) are the setting laws of $\gamma$, and the PID adaptive adjustment of $\gamma$ is based on the control of constraint violation $g$ and is gradient-falling. Figure 3 illustrates that $\lambda_{1} \ldots \lambda_{5}$ are the PID adaptive curves for the constrained term, 


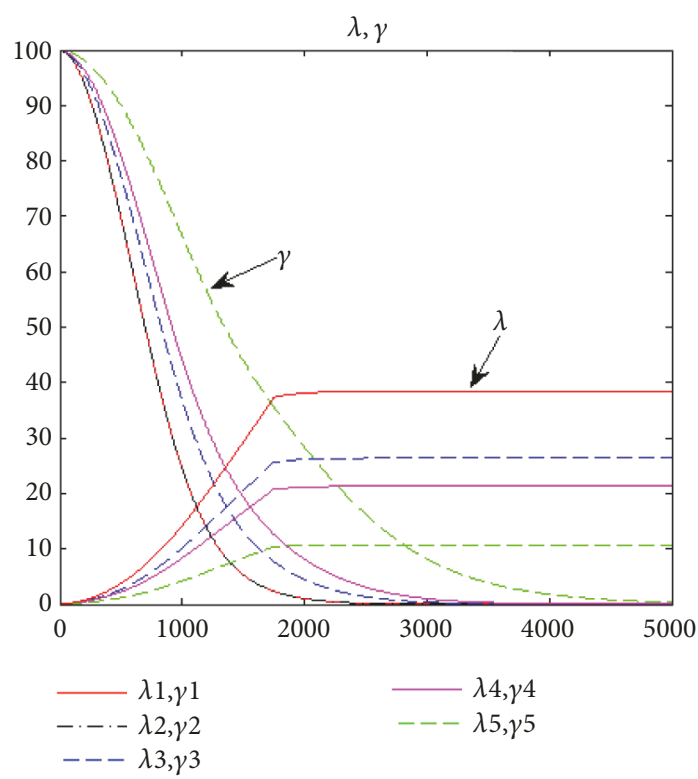

Figure 3: Adaptive adjustment curves for $\lambda$ and $\gamma$.

and $\gamma_{1} \ldots \gamma_{5}$ are the PID adaptive curves for the objective term.

The adaptive parameter setting can often obtain good optimization effect. However, the PID parameters control may have conflict with the trend of gradient descending; when the two kinds of adjustments conflict on some equilibrium point, oscillations are triggered. Thus the network cannot converge to balance point stably. Moreover, the adaptive parameter setting is bounded. Thus, when parameter adjustment reaches the upper or the lower limit, adjustment strength does not change any more, thereby impeding the network converging to the global optimum. Basing on above difficulties, the further strategy for improvement is proposed.

3.6. Efficiency Factor Strategy. As a problem of the optimal solutions distributing on boundary of constraints, the adjustments of constrained terms sometimes conflict with the adjustment of objective terms, which may cause oscillations on equilibrium point. In addition, as the analysis in 3.5, the PID parameter adjustments may violate the gradient descent trend of the network, which also leads to oscillations on balance point. The main reason triggering oscillations is that the constraint parameter controls and the objective parameter controls are hard to balance. For example, when the constrained term dominates the descent, energy loss descends toward constraints satisfaction, whereas the optimization objective might not be satisfied. Therefore, in the next iteration, regulating strength for the objective term grows larger; thus the objective term dominates the descent and drives it toward the optimum, whereas the convergence might break away the domain of feasible solution and the constraints might not be satisfied. Such repeated processes lead to oscillations on the equilibrium point.

To solve the problem, one strategy is to convert the constrained optimization to multiobjective optimization.

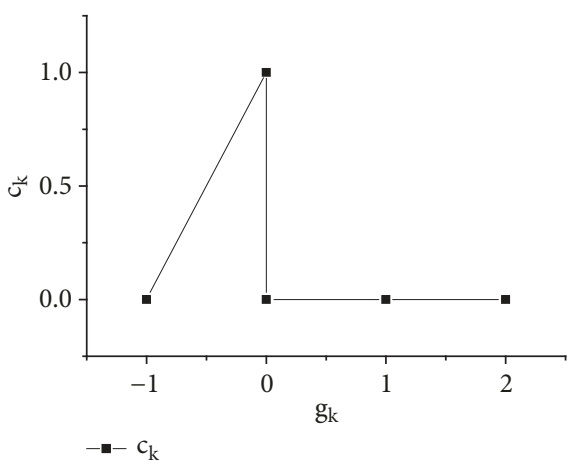

FIGURE 4: Curve of efficiency factor $c_{k}$.

However, the higher the dimension of the multiobjective optimization, the more difficult the optimization model is to be calculated [22]. Therefore, this study takes another way that introduces an efficiency factor into the energy function to prioritize the constrained terms over the objective term.

The second term of (7) is the term for associated constraints. $c_{k}$ is the efficiency factor of Section $k, k=1 \ldots n$. $g_{k}$ is the constraint violation of Section $k$, which belongs to $[-1,0]$. If Section $k$ is fully retracted, then the cylinder has full travel allowance and " 1 " for driving Section $k, g_{k}$ will be -1 . If Section $k$ is fully extended, then the cylinder has no travel allowance, " 0 " for driving Section $k$, and $g_{k}$ will be 0 . Generally, $c_{k}$ should be normalized, but because $g_{k}$ has been limited in the range of $[-1,0]$ and $c_{k}$ has been limited to $[0,1]$ thereby $c_{k}$ does not need to be normalized here:

$$
\begin{aligned}
c_{k} & =0, \quad g_{k}>0 ; \\
c_{k} & =1+g_{k}, \quad g_{k} \leq 0 . \\
c & =\prod_{k=1}^{n} c_{k}
\end{aligned}
$$

Equation (20) indicates that when $g_{k}>0$, convergence is beyond the feasible domain, and then $c_{k}=0$. When $-1 \leq g_{k} \leq$ 0 , convergence is within the feasible domain, and then $c_{k}$ is variable with $g_{k}$ changing. When $g_{k}=0$, network converges to the boundary of the feasible domain, which is the optimal solution, and then $c_{k}=1$. Figure 4 shows the curve of $c_{k} . c$ is the multiplication of $c_{k}$ as presented in (21).

\section{Simulation, Analysis, and Discussion}

4.1. Effect of TPO. Let $n=5, m=4$ in (7); that is, a SPMB has five telescopic sections, and four holes arranged on each section. The holes locations are the same for all sections. Parameters are set as shown in Table 2. Input the initial state $A=\left[\begin{array}{llll}2 & 2 & 2 & 2\end{array}\right.$ 2] and the target state $T=\left[\begin{array}{llll}2 & 1 & 2 & 1\end{array}\right]$ and run the TPO HNN program to search for RBC state $V$.

Figure 5 shows the telescoping processes with four RBC states. In these figures, the color blocks represent the sections, and the blue dotted line is the single-cylinder travel "1." Because the travel of cylinder driving section $\left(S_{\text {boom }}\right)$ can 
TABLE 2: Parameter setting of simulations.

\begin{tabular}{|c|c|c|c|c|}
\hline HNN & $\lambda$ of PID & $\gamma$ of PID & Momentum & Adagrad \\
\hline$B=5$ & $B=5$ & $B=5$ & $B=5$ & $B=5$ \\
\hline$\mu=0.0001$ & $\mu_{\lambda}=0.0001$ & $\mu_{\gamma}=0.0001$ & $l_{r}=0.001$ & $l_{r}=0.1$ \\
\hline$\lambda=\left[\begin{array}{lllll}5 & 5 & 5 & 5 & 5\end{array}\right]$ & $K_{p \lambda}=10$ & $K_{p \gamma}=0.1$ & $\lambda=\left[\begin{array}{lllll}5 & 5 & 5 & 5 & 5\end{array}\right]$ & $\lambda=\left[\begin{array}{lllll}5 & 5 & 5 & 5 & 5\end{array}\right.$ \\
\hline \multirow[t]{3}{*}{$\gamma=\left[\begin{array}{lllll}5 & 5 & 5 & 5 & 5\end{array}\right]$} & $K_{i \lambda}=0.1$ & $K_{i \gamma}=0.01$ & $\gamma=\left[\begin{array}{lllll}1 & 5 & 5 & 5 & 5\end{array}\right]$ & $\gamma=\left[\begin{array}{lllll}5 & 5 & 5 & 5 & 5\end{array}\right.$ \\
\hline & $K_{d \lambda}=1$ & $K_{d \gamma}=1$ & $\mu_{m}=0.9$ & \\
\hline & $\lambda(0)=0$ & $\gamma(0)=1000$ & & \\
\hline
\end{tabular}
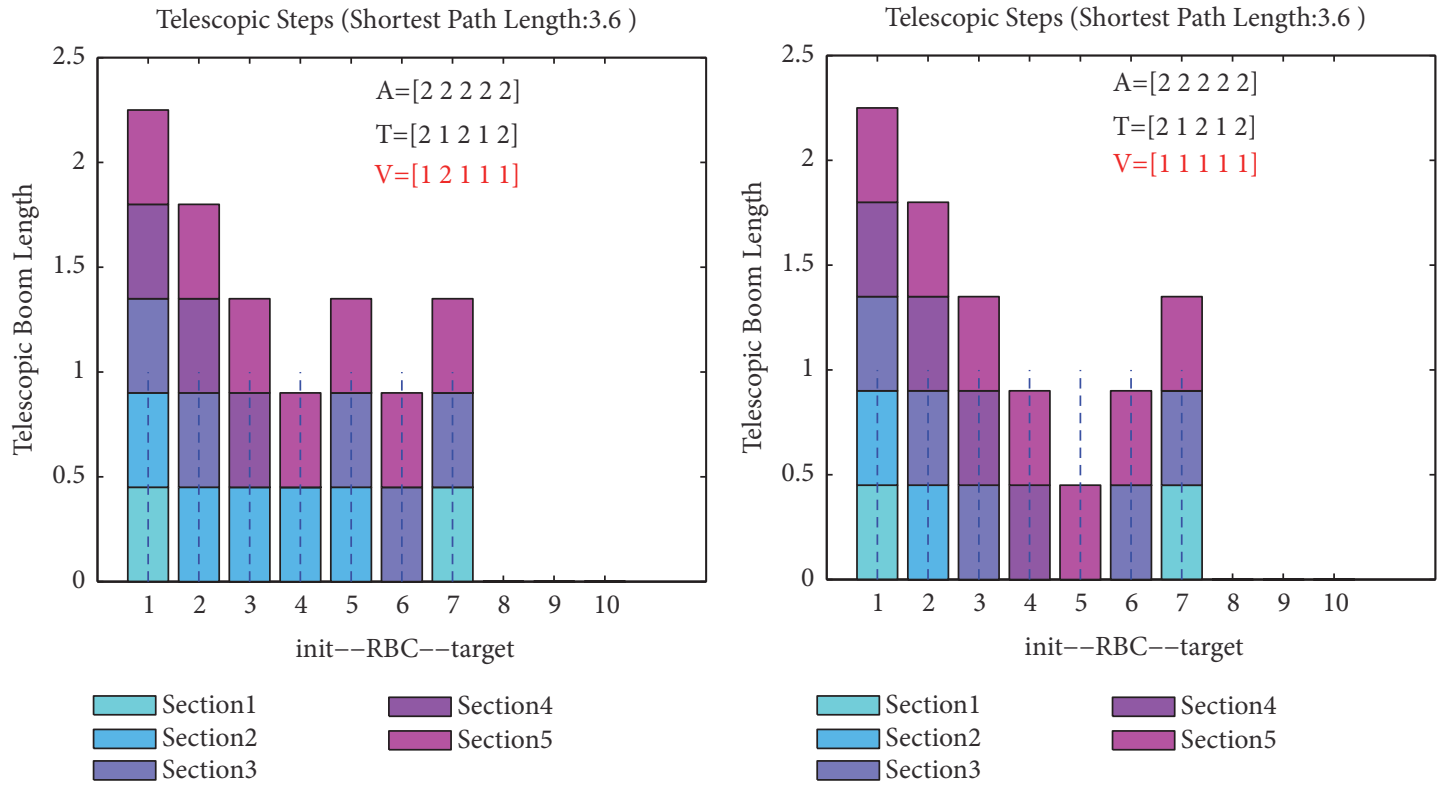

(a) $V=\left[\begin{array}{lllll}1 & 2 & 1 & 1 & 1\end{array}\right]$

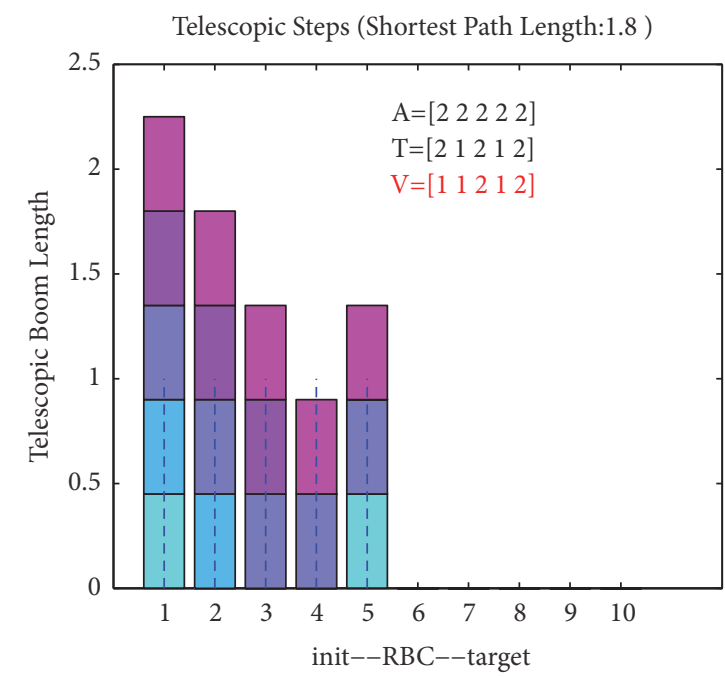

(b) $V=\left[\begin{array}{llllll}1 & 1 & 1 & 1 & 1\end{array}\right]$
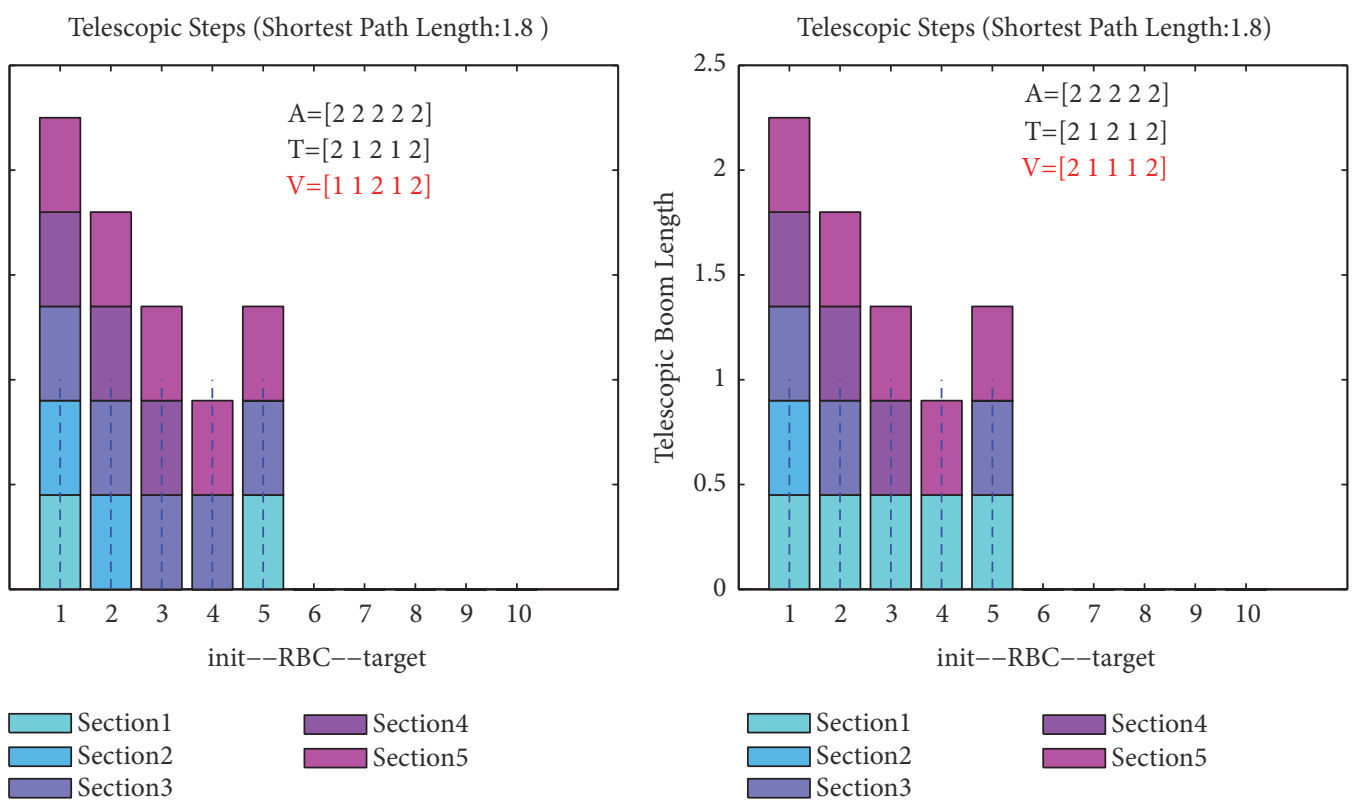

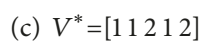

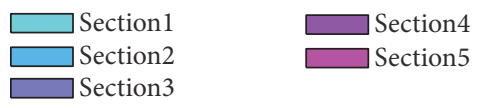

(d) $V^{*}=\left[\begin{array}{lllll}2 & 1 & 1 & 1 & 2\end{array}\right]$

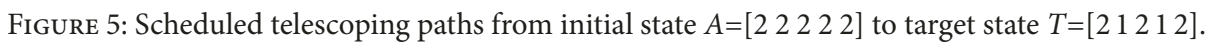


TABLE 3: Test results for TPO-HNN model.

\begin{tabular}{|c|c|c|c|c|c|c|c|c|c|c|c|c|}
\hline data & $\mathrm{HN}$ & & HNN & & $\mathrm{HNN}+$ & ID & $\mathrm{HNN}+\mathrm{P}$ & $\mathrm{D}+c$ & $\mathrm{HNN}+\mathrm{M}$ & entum & $\mathrm{HNN}+\mathrm{A}$ & agrad \\
\hline Run 25 times & $\begin{array}{l}\text { Optimal/ } \\
\text { valid }\end{array}$ & time & $\begin{array}{l}\text { Optimal/ } \\
\text { valid }\end{array}$ & time & $\begin{array}{l}\text { Optimal/ } \\
\text { valid }\end{array}$ & time & $\begin{array}{l}\text { Optimal/ } \\
\text { valid }\end{array}$ & time & $\begin{array}{l}\text { Optimal/ } \\
\text { valid }\end{array}$ & time & $\begin{array}{l}\text { Optimal/ } \\
\text { valid }\end{array}$ & time \\
\hline $\begin{array}{l}\mathrm{A}=\left[\begin{array}{lllll}2 & 2 & 2 & 2 & 2\end{array}\right] \\
\mathrm{T}=\left[\begin{array}{lllll}2 & 1 & 2 & 1 & 2\end{array}\right]\end{array}$ & $7 / 19$ & 0.511 & $13 / 25$ & 0.499 & $12 / 22$ & 1.043 & $10 / 21$ & 1.119 & $5 / 20$ & 0.535 & $3 / 22$ & 0.554 \\
\hline $\begin{array}{l}\mathrm{A}=\left[\begin{array}{lllll}3 & 2 & 3 & 2 & 1\end{array}\right] \\
\mathrm{T}=\left[\begin{array}{lllll}1 & 2 & 2 & 2 & 2\end{array}\right]\end{array}$ & $1 / 7$ & 0.531 & $3 / 20$ & 0.528 & $1 / 15$ & 1.081 & $5 / 14$ & 1.082 & $2 / 6$ & 0.590 & $3 / 7$ & 0.510 \\
\hline $\begin{array}{l}\mathrm{A}=\left[\begin{array}{lllll}2 & 1 & 3 & 3 & 1\end{array}\right] \\
\mathrm{T}=\left[\begin{array}{lllll}1 & 2 & 2 & 2 & 2\end{array}\right]\end{array}$ & $2 / 5$ & 0.553 & $6 / 20$ & 0.549 & $5 / 13$ & 1.064 & $5 / 19$ & 1.164 & $4 / 4$ & 0.537 & $1 / 5$ & 0494 \\
\hline $\begin{array}{l}\mathrm{A}=\left[\begin{array}{lllll}1 & 2 & 4 & 2 & 1\end{array}\right] \\
\mathrm{T}=\left[\begin{array}{lllll}2 & 2 & 2 & 2 & 2\end{array}\right]\end{array}$ & $2 / 10$ & 0.565 & $6 / 25$ & 0.551 & $5 / 17$ & 1.120 & $7 / 20$ & 1.103 & $3 / 6$ & 0.526 & $5 / 11$ & 0.517 \\
\hline $\begin{array}{l}\mathrm{A}=\left[\begin{array}{lllll}2 & 1 & 2 & 1 & 2\end{array}\right] \\
\mathrm{T}=\left[\begin{array}{lllll}2 & 3 & 2 & 2 & 1\end{array}\right]\end{array}$ & $1 / 15$ & 0.530 & $8 / 24$ & 0.516 & $5 / 15$ & 1.024 & $10 / 14$ & 1.145 & $4 / 17$ & 0.533 & $3 / 15$ & 0.526 \\
\hline $\begin{array}{l}\text { Average } \\
\text { percentage }\end{array}$ & $\begin{array}{l}10 \% / \\
45 \%\end{array}$ & & $\begin{array}{l}29 \% / \\
95 \%\end{array}$ & & $\begin{array}{c}22 \% / \\
65 \%\end{array}$ & & $\begin{array}{c}30 \% / \\
70 \%\end{array}$ & & $\begin{array}{l}14 \% / \\
42 \%\end{array}$ & & $\begin{array}{l}12 \% / \\
48 \%\end{array}$ & \\
\hline
\end{tabular}

identify path length well, we use $S_{\text {boom }}$ instead of the total travel of cylinder $\left(S_{\text {total }}\right)$ to indicate the optimization effect.

If the RBC states are not well optimized or not optimized, the telescoping paths will require six steps, and $S_{\text {boom }}$ will be 3.6, as shown in Figures 5(a) and 5(b). If the RBC states are well optimized, they will just require four steps, and $S_{\text {boom }}$ will only be 1.8, as shown in Figures 5(c) and 5(d). The efficiency of boom length changing increases obviously after well optimization.

4.2. Improvement of the TPO HNN Model. The probability that energy loss converges to the saddle point is large because most natural objective functions have exponential saddle points. Such points are unstable, implying invalid solutions. Momentum strategy helps accelerate gradient descent in certain directions and suppress oscillation [23].

$$
\begin{aligned}
& v(t)=\mu_{m} \times v(t-1)+l_{r} \times d u(t-1) \\
& u(t)=u(t-1)-v(t)
\end{aligned}
$$

The given formula is the iterative formula of momentum strategy, where $v(t-1)$ is the direction of the last iteration, $\mu_{m}$ is the momentum coefficient, $l_{r}$ is the learning rate (iteration step size), $d u(t)$ is the gradient, and $u(t)$ is the output [24].

The descent can easily stuck in the saddle point, thereby leading to divergent oscillation with a fixed learning rate. The adaptive gradient algorithm (Adagrad) adaptively adjusts the learning rate. $\varepsilon$ is a very small constant that prevents the denominator from becoming zero [25].

$$
\begin{aligned}
\operatorname{acc}(t) & =\operatorname{acc}(t-1)+(d u(t-1))^{2} \\
u(t) & =u(t-1)-\frac{l_{r}}{\sqrt{a c c(t)+\varepsilon}} d u(t-1)
\end{aligned}
$$

In order to improve the performance of TPO model, these two strategies are used in TPO model to compare the effects with PID strategy and efficiency factor strategy. In the following, six strategies are simulated, respectively: HNN, HNN with efficiency factor, HNN with PID adjustment, HNN with PID and efficiency factor mixed, HNN with momentum, and HNN with Adagrad. Five groups of data are tested, and each group runs 25 times. The iteration is 2,000 , and $V_{0}$ is randomly generated. Parameter settings are as Table 2, and the results are listed in Table 3.

When parameters are set as constants, the performance of HNN model is not good. During the 25 runs of program, the obtained valid solutions percentage is lower than $50 \%$. However, after introducing efficiency factor in the HNN model, the performance is evidently improved, and the obtained average valid solution percentage increases to $95 \%$. The PID adaptive parameter adjustment strategy also performs well, and the obtained average valid solution percentage reaches $65 \%$. The HNN with both PID and efficiency factor strategy also improves the results well, and the obtained average valid solution percentage is $70 \%$.

Furthermore, the average optimal solution percentages obtained by the efficiency factor strategy, by the PID strategy, and by the PID and efficiency factor mixed strategy are the best. Thus either the PID strategy or the efficiency factor strategy is effective in promoting the solution qualities.

As a problem that its high-quality solutions are scattered on the boundary of a feasible domain as Keane's bump problem [26], the oscillations of TPO model are caused by the mutual conflicts of the constrained terms and the objective terms. Besides, the PID adaptive parameters control might have contradiction with the trend of gradient descending, which brings the convergence oscillations as well while the efficiency factor strategy is workable to balance the contradictions of the constrained terms and the objective terms.

Although the strategy of momentum and the strategy of changing learning rate are efficient to help the convergence escape saddle point, they do not work well here, since stopping at saddle point is not the reason of triggering the oscillations while TPO model converging. 


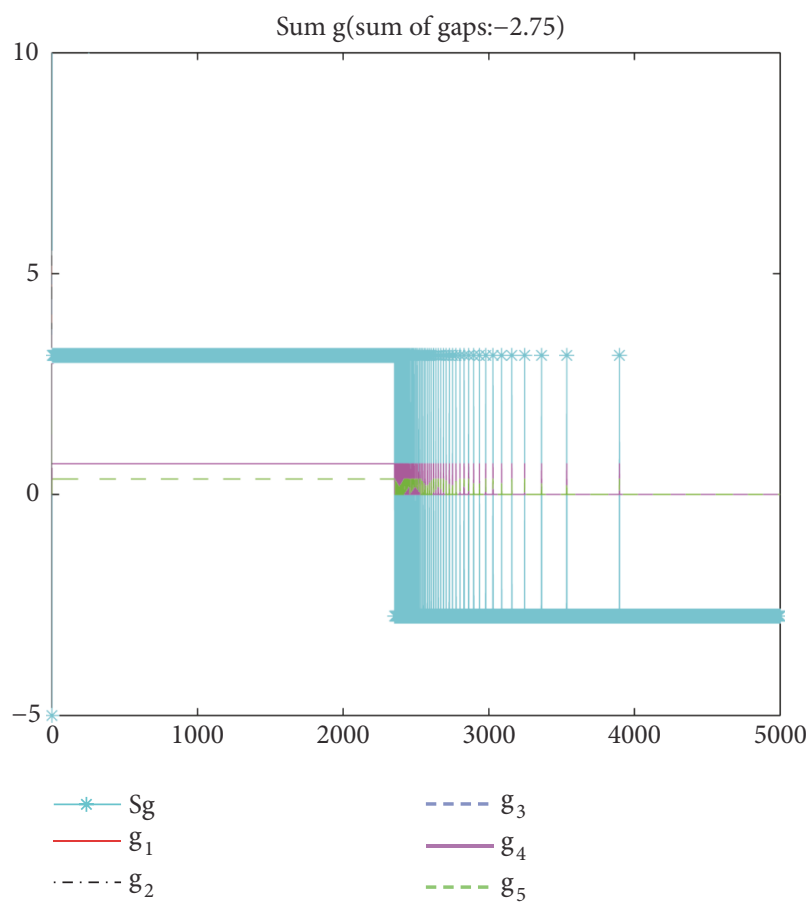

(a) $g_{\mathrm{k}}$ control of HNN+PID strategy

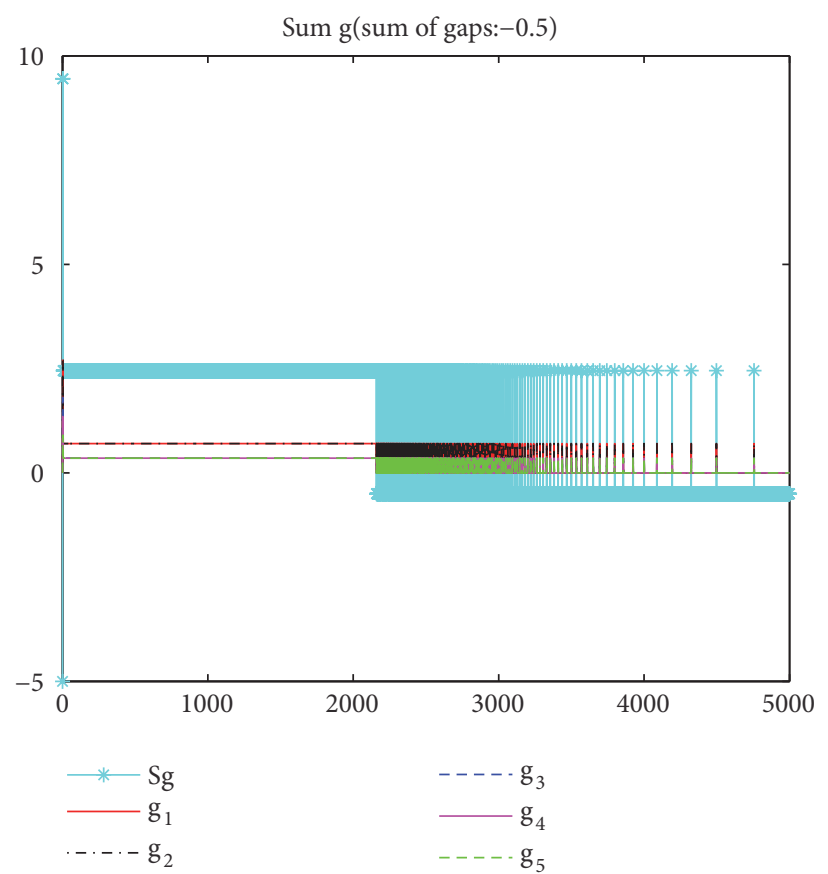

(b) $g_{\mathrm{k}}$ control of HNN+PID+c strategy

FIGURE 6: The constraint violation $(g)$ changes during convergence process.

In addition, PID adaptive parameter adjustment strategy has a slightly higher complexity than other methods. Thus, its calculation time is slightly longer than other methods.

4.3. Analysis of TPO Calculation Process. The efficiency factor strategy plays an important role in promoting the qualities of solutions derived from Table 3. Through comparing the convergences before and after efficiency factor strategy being added in the model, we can know how the efficiency factor strategy works.

The parameter settings are as in Table 2, the iteration is 5,000 , the initial $\mathrm{RBC}\left(V_{0}\right)$ is fixed, and the same initial conditions are applied for the compared strategies. Test data are $A=\left[\begin{array}{lllll}2 & 1 & 3 & 3 & 1\end{array}\right], T=\left[\begin{array}{lllll}1 & 2 & 2 & 2 & 2\end{array}\right]$. HNN with PID adaptive parameters, HNN with PID adaptive parameters and efficiency factor mixed, HNN with constant parameters and HNN with efficiency factor-these four strategies-are tested.

HNN PID strategy adaptively adjusts constrained parameter $\lambda$ and objective parameter $\gamma$ by controlling the constraint violation $g$ to close the zero which is the boundary of the feasible domain. $g_{1} \ldots g_{5}$ are the constraint violations of the five sections, and $S_{g}$ is the sum of $g_{1} \ldots g_{5}$. Figure 6(a) depicts the $g_{1} \ldots g_{5}$ and $S_{9}$ changes in the process of the HNN PID network converging with $V_{0}$ as the initial input. When the iteration is over, $S_{g}$ obtains high cylinder travel allowance, -2.75 , which indicates that the path is not fully optimized. Figure 6(b) demonstrates the $g_{1} \ldots g_{5}$ and $\mathrm{S}_{\mathrm{g}}$ changes in the process of the HNN PID and efficiency factor mixed network converging with the same $V_{0}$ as the initial input. When the iteration is over, $S_{g}$ obtains little cylinder travel allowance, -0.5 , which indicates that the path is well optimized when the efficiency factor is being added in the HNN PID model.

Figures 7(a) and 7(b) compare the solution V's convergence process under the given conditions. Figure 7 (a) shows that $V_{4}$ has oscillations triggered between "1" and "2" after the program iterates approximately 2,500 times. The reason is that when iterating to a certain extent, value of $V_{41}$ and value of $V_{42}$ are almost equal, and the definition of matrix $V$ is based on a "maximum pick" principle where the maximum value among the row elements is picked out as the output, and all else values are ignored. Thus, when two similar elements exist in the same row, a selection jumps between these two elements. Figure 7(b) shows that when efficiency factor is introduced into the PID strategy, the oscillations of $V_{4}$ do not happen, and it converges stably on " 2 ." The efficiency factor prioritizes the constrained terms over the objective terms and all the terms do not have to compete with each other. Thus, the oscillations are suppressed and the generations of high quality solutions are increased.

Figure 8(a) describes the scheduled telescoping path in

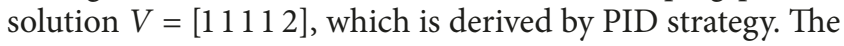
process requires eight steps from the initial state to the RBC state and then to the target state. $S_{\text {boom }}$ is 4.05 . Figure $8(\mathrm{~b})$ illustrates the scheduled telescoping path in solution $V=[1$ $\left.\begin{array}{lll}1 & 2 & 2\end{array}\right]$, which is derived by PID and efficiency factor mixed strategy. The process requires seven steps from the initial state to the RBC state and then to the target state. $S_{\text {boom }}$ is 3.15 . The efficiency factor strategy is useful to help the convergence to optimal solution. 

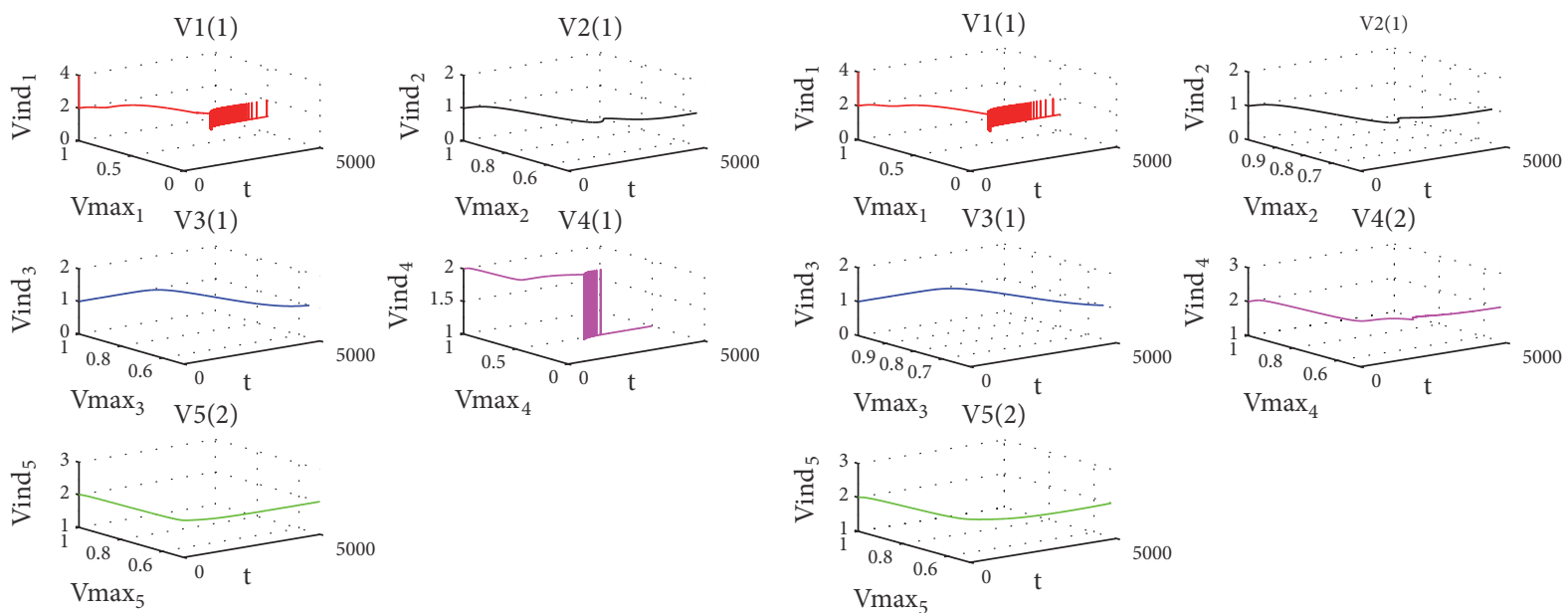

(a) $V$ convergence of HNN+PID

(b) $V$ convergence of $\mathrm{HNN}+\mathrm{PID}+\mathrm{c}$

FIGURE 7: The RBC $V$ converging processes.

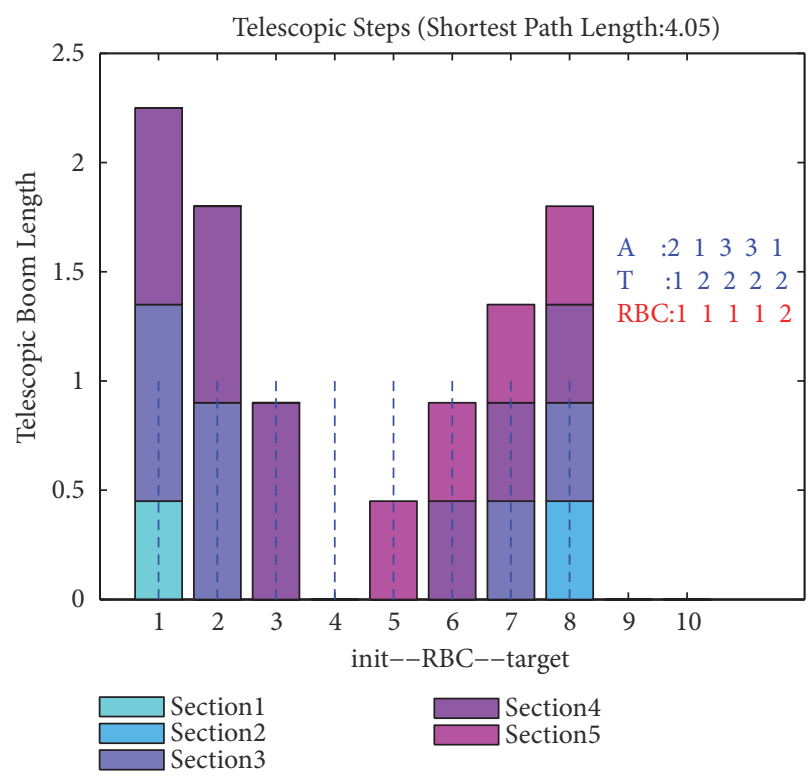

(a) With HNN+PID strategy

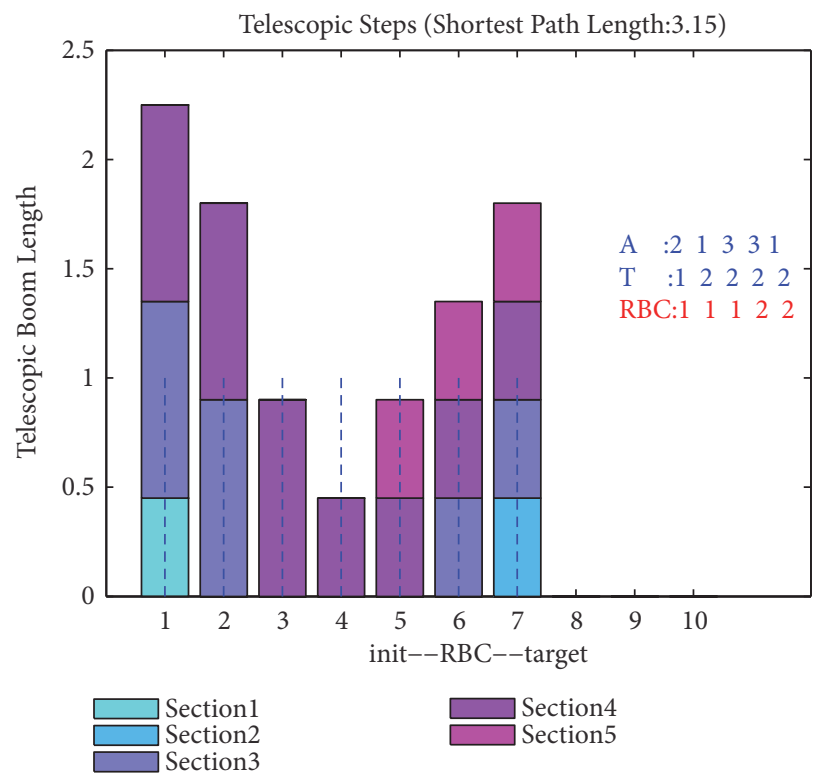

(b) With HNN+PID+c strategy

FIGURE 8: Scheduled telescoping path from initial state $A=\left[\begin{array}{lllll}2 & 1 & 3 & 3 & 1\end{array}\right]$ to target state $T=\left[\begin{array}{llll}1 & 2 & 2 & 2\end{array}\right]$.

Figure 9 demonstrates the energy loss curves with PID strategy before and after introducing in efficiency factor under the same initial conditions. Blue curve is the energy loss of PID strategy, which converges to minimum 10.482, corresponding to a valid solution $V=\left[\begin{array}{lllll}1 & 1 & 1 & 1 & 2\end{array}\right]$. Red curve is the energy loss of PID mixed with efficiency factor strategy, which converges to minimum 5.9212, corresponding to an optimal $V=\left[\begin{array}{llll}1 & 1 & 1 & 2\end{array}\right]$ 2]. The comparison indicates that efficiency factor is helpful in converging to the optimal point.

Figure 10 demonstrates the energy loss curves of HNN with constant parameters strategy before and after introducing efficiency factor under the same initial conditions. Blue curve is the energy loss, which converges to minimum 6.5127, corresponding to an invalid solution $V=\left[\begin{array}{llll}1 & 2 & 2 & 1\end{array}\right]$. Red curve is the energy loss with efficiency factor strategy, which converges to minimum 0.30891 , corresponding to an optimal $V=\left[\begin{array}{llll}1 & 1 & 1 & 2\end{array}\right]$ 2]. The comparison indicates that the efficiency factor greatly enhances the descent searching strength, thus making the energy loss converging to a global minimum. While having no efficiency factor strategy, the energy loss should have oscillated around an invalid solution.

In summary, both PID strategy and efficiency factor strategy are effective in improving the performance of the TPO model. The efficiency factor strategy has its advantages on balancing the conflicts between the constrained terms and objective terms, so it can lead to stable convergence efficiently and can get high quality solutions thereby. 


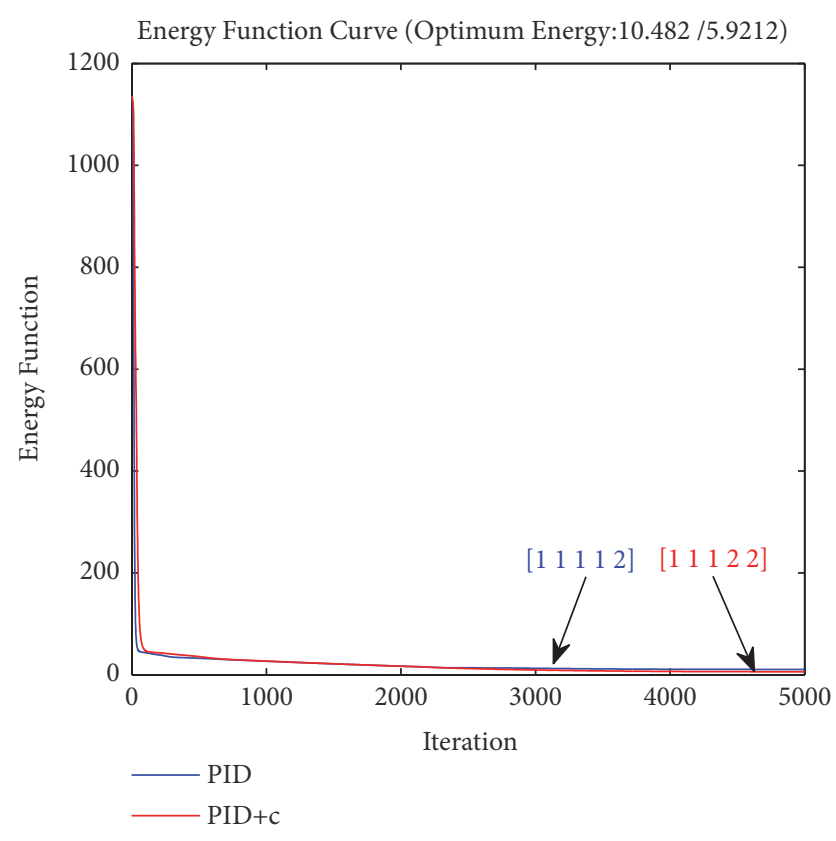

Figure 9: Energy curves of HNN+PID and HNN+PID+c.

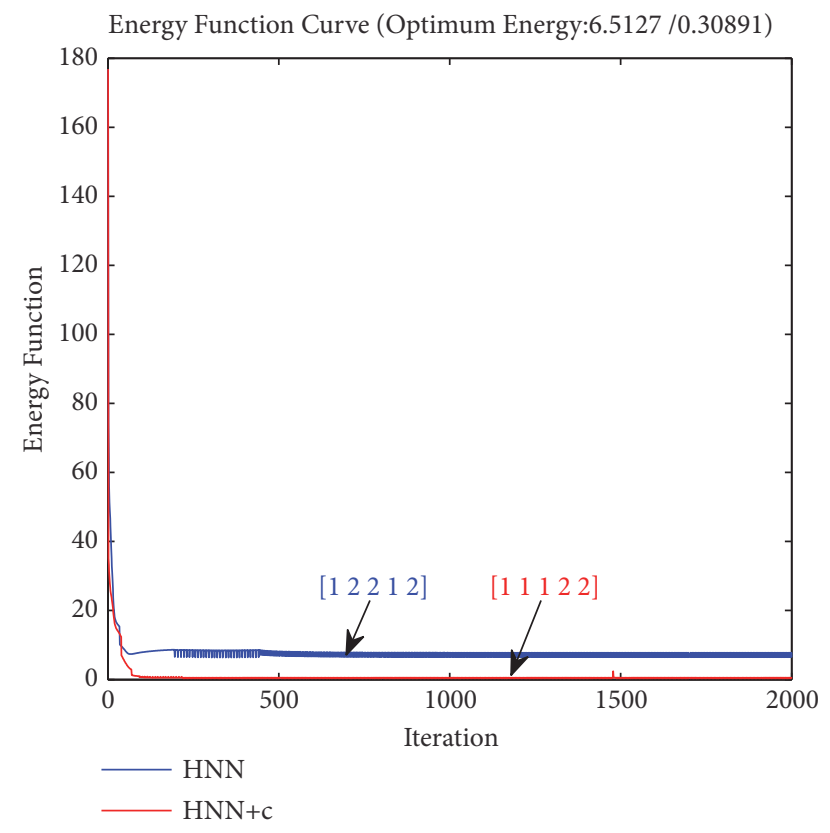

FIGURE 10: Energy curves of HNN and $\mathrm{HNN}+\mathrm{c}$.

\section{Conclusion and Prospect}

This study investigates the practical engineering optimization problem, TPO of SPMB. TPO aims to obtain the maximum RBC. During the SPMB telescoping process, the movement of each section is associated with each other and mutually restrained; that is, multiple associated constraints are involved in TPO problem. TPO is a strongly constrained problem with the optimal solutions scattered on constraint boundaries. In a word, TPO aims to obtain the largest RBC; thus the scheduled telescoping steps can be the least and the cylinder telescoping paths can be the shortest when SPMB changes its boom length.

The energy function model of HNN can mitigate the complexity of constraints processing; thus, this article constructs the TPO mathematical model in quadric penalty function form of HNN. In the energy function, seeing that determining the penalty parameters is difficult, a PID strategy is proposed that adaptively adjusts the penalty parameters $\lambda$ of the constrained term and the penalty parameters $\gamma$ of the optimization objective term by controlling constraint violation value $g_{k}$. Moreover, the $\lambda$ is set as gradient-rising and the $\gamma$ is set as gradient-falling according to the trend of the dynamic equation gradient descending. The overlapping region of $\lambda$ and $\gamma$ is the place for the optimization searching of the network, where the larger the optimization searching area, the more likely converging to high-quality solution.

TPO belongs to the optimization category that optimal solutions scattered on boundary of feasible domain. The constrained terms and the objective terms are sometimes mutually exclusive, so they are difficult to be balanced. The oscillations being triggered sometimes on equilibrium point make the network hardly converge to global optimal. Beside, although the PID strategy is effective in driving the convergence towards the valid or the optimal solutions, the PID adaptive parameters control might have contradiction with the trend of gradient descending, which brings the oscillations on equilibrium point as well. Oscillation is the reason that the network could not converge efficiently.

The introduction of efficiency factor that prioritizes the constrained terms over the objective terms is efficient to solve the oscillation problem and gets the best generations of both valid and optimal solutions.

The simulation result shows that compared with the boom length changing before optimization, both the number of sections that need to be moved (scheduled telescoping steps) and the total travels of the cylinder can be reduced by 10\%-30\% after optimization.

Compared to the effect of the HNN with constant parameters strategy, the HNN PID strategy can improve the valid solutions percentage from $50 \%$ to $65 \%$ while the $\mathrm{HNN}$ efficiency factor strategy is more efficient and improves the valid solutions percentage from $50 \%$ to $95 \%$. The optimal solutions percentage obtained by efficiency factor strategy is the highest as well. In summary, the efficiency factor strategy is excellent at balancing the conflicts between the constrained terms and objective terms.

The PID strategy and the efficiency factor strategy are universal that can be applied for other problems solving. The HNN method for TPO problem is a universal model that can be applied to calculate the TPOs under various conditions; for example, the boom sections $n$, the holes number $m$, and the holes location combination $d$ on each section are variable, not limited by $n=5$ and $m=4, d=\left[\begin{array}{llll}0 & 0.45 & 0.9 & 1\end{array}\right]$ of the example being illustrated in the article. In addition, the HNN method can be used to the TPOs of complex SPMB mechanisms; for example, the holes number and the holes locations are various for every section. 
Although the HNN model with PID strategy and the HNN model with efficiency factor strategy both obtain good results, there are flaws that should be attended,

(I) PID adaptive parameters adjustment strategy has to determine the proportional coefficient $K_{p}$, the integral coefficient $K_{i}$, and the differential coefficient $K_{d}$; thus it is not the fully self-adaptive strategy.

(II) The searching algorithm basing on gradient descent method is hard to obtain $100 \%$ valid solutions, and the generation of optimal solutions is not high generally.

In future, the genetic algorithm (GA) could be used to calculate TPOs, seeing that GA only requires the optimized objectives being computable, and no need being continuous and differentiable; besides, GA is based on population evolution that converges to global optimums, so it can overcome the weaknesses of traditional algorithms based on gradient optimization.

\section{Data Availability}

The [DATA programs] data used to support the findings of this study are available from the corresponding author upon request.

\section{Conflicts of Interest}

The authors declare that they have no conflicts of interest.

\section{References}

[1] C. Zhan, Q. Liu, J. Guo et al., "Single-cylinder pin-type telescopic boom track optimized control method and control system thereof," Tech. Rep. WO/2011/038633, 2011.

[2] Y. Mao and K. Chen, "Sequential telescopic path optimization method of single-cylinder pin-type multi-section boom," Tech. Rep. CN106744386B, 2018 (Chinese).

[3] Y. Mao and K. Chen, "An efficient sequential telescopic path optimization method of single-cylinder pin-type multi-section boom," Tech. Rep. CN106744389B, 2018 (Chinese).

[4] U.-P. Wen, K.-M. Lan, and H.-S. Shih, "A review of Hopfield neural networks for solving mathematical programming problems," European Journal of Operational Research, vol. 198, no. 3, pp. 675-687, 2009.

[5] J. J. Hopfield, "Neural networks and physical systems with emergent collective computational abilities," Proceedings of the National Acadamy of Sciences of the United States of America, vol. 79, no. 8, pp. 2554-2558, 1982.

[6] J. J. Hopfield and D. W. Tank, "Neural computation of decisions in optimization problems," Biological Cybernetics, vol. 52, no. 3 , pp. 141-152, 1985.

[7] B. Shirazi and S. Yih, "Critical analysis of applying Hopfield neural net model to optimization problems," in Proceedings of the IEEE International Conference on Systems, Man and Cybernetics, vol. 1, pp. 210-215, 1989.

[8] S. Aiyer, M. Niranjan, and F. Fallside, "A theoretical investigation into the performance of the Hopfield model," IEEE Transactions on Neural Networks and Learning Systems, vol. 1, no. 2, pp. 204-215, 1990.
[9] S. Abe, "Global convergence and suppression of spurious states of the Hopfield neural networks," IEEE Transactions on Circuits and Systems I: Fundamental Theory and Applications, vol. 40, no. 4, pp. 246-257, 1993.

[10] S. Shouyu and J. Zheng, "A modified algorithm and theoretical analysis for hopfield network solving TSP," Acta Electronica Sinica, 1995 (Chinese).

[11] J. Zhang, "Theoretical analysis and improvement of the neural network to solve the TSP problem," Journal of Xidian University, vol. 23, 1996 (Chinese).

[12] P. M. Talaván and J. Yáñez, "Parameter setting of the Hopfield network applied to TSP," Neural Networks, vol. 15, no. 3, pp. 363373, 2002.

[13] S. Effati and M. Baymani, "A new nonlinear neural network for solving convex nonlinear programming problems," Applied Mathematics and Computation, vol. 168, no. 2, pp. 1370-1379, 2005.

[14] S. Effati and M. Jafarzadeh, "A new nonlinear neural network for solving a class of constrained parametric optimization problems," Applied Mathematics and Computation, vol. 186, no. 1, pp. 814-819, 2007.

[15] S. Ruder, "An overview of gradient descent optimization algorithms," 2016.

[16] R. Wang, S. Guo, and K. Okazaki, "A hill-jump algorithm of Hopfield neural network for shortest path problem in communication network," Soft Computing, vol. 13, no. 6, pp. 551-558, 2009.

[17] L. Wang and F. Tian, "Noisy chaotic neural networks for solving combinatorial optimization problems," in Proceedings of the IEEE-INNS-ENNS International Joint Conference on Neural Networks (IJCNN '00), vol. 4, pp. 37-40, IEEE, Como, Italy, 2000.

[18] L. Perko, Differential Equations and Dynamical Systems, Springer, 2001.

[19] R. Ge, F. Huang, C. Jin, and Y. Yuan, "Escaping from saddle points: online stochastic gradient for tensor decomposition," Journal of Machine Learning Research, vol. 40, no. 2015, 2015.

[20] A. El-Bouri, S. Balakrishnan, and N. Popplewell, "A neural network to enhance local search in the permutation flowshop," Computers \& Industrial Engineering, vol. 49, no. 1, pp. 182-196, 2005.

[21] H. Qu, Z. Yi, and H. Tang, "Improving local minima of columnar competitive model for TSPs," IEEE Transactions on Circuits and Systems I: Regular Papers, vol. 53, no. 6, pp. 13531362, 2006.

[22] Y. Wang, Z. Cai, G. Guo, and Y. Zhou, "Multiobjective optimization and hybrid evolutionary algorithm to solve constrained optimization problems," IEEE Transactions on Systems, Man, and Cybernetics, Part B: Cybernetics, vol. 37, no. 3, pp. 560-575, 2007.

[23] M. Nielsen, "Neural networks and deep learning," 2016. http:// neuralnetworksanddeeplearning.com/.

[24] N. Qian, "On the momentum term in gradient descent learning algorithms," Neural Networks, vol. 12, no. 1, pp. 145-151, 1999.

[25] J. Duchi, E. Hazan, and Y. Singer, "Adaptive subgradient methods for online learning and stochastic optimization," Journal of Machine Learning Research, vol. 12, no. 7, pp. 257-269, 2011.

[26] M. Schoenauer and Z. Michalewicz, "Boundary operators for constrained optimization problems," in Proceedings of the 7th International Conference on Genetic Algorithms, T. Baeck, Ed., pp. 322-329, Morgan Kaufmann Publishers, San Mateo, Calif, USA, 1997. 


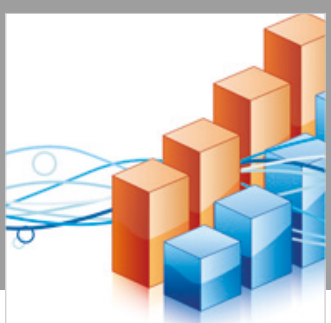

Advances in

Operations Research

\section{-n-m}
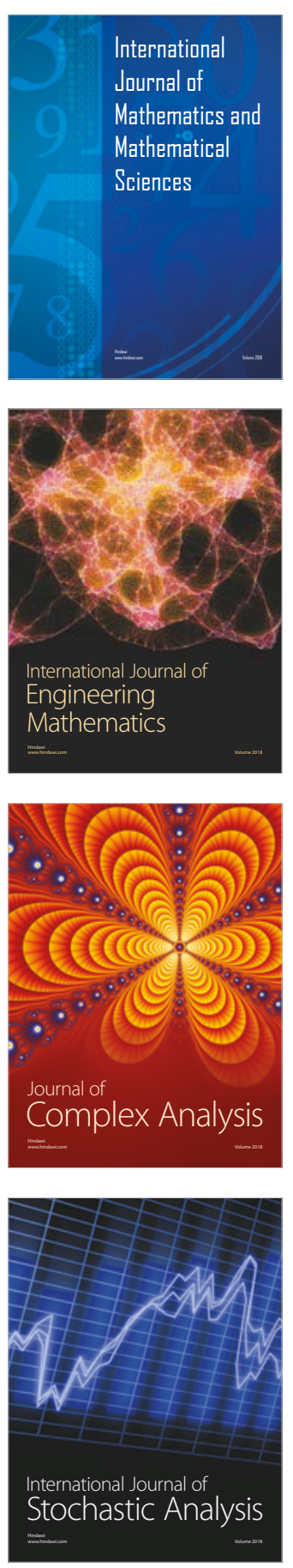
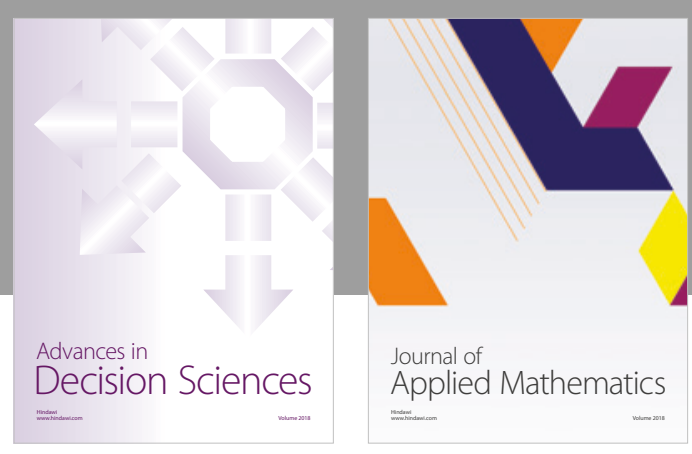

Journal of

Applied Mathematics
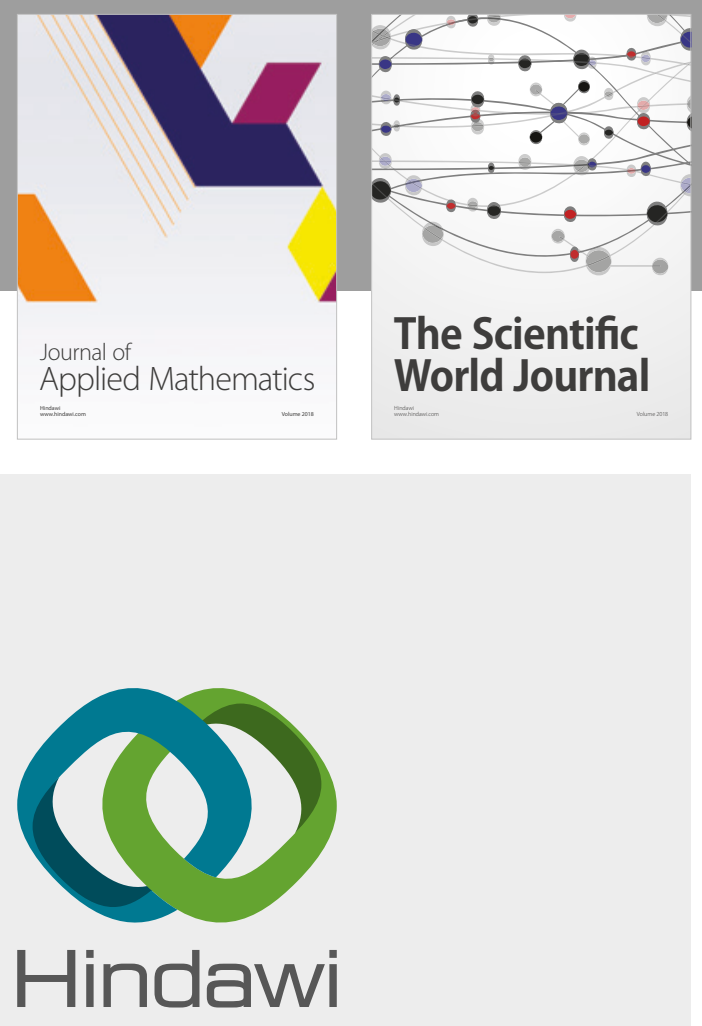

Submit your manuscripts at

www.hindawi.com

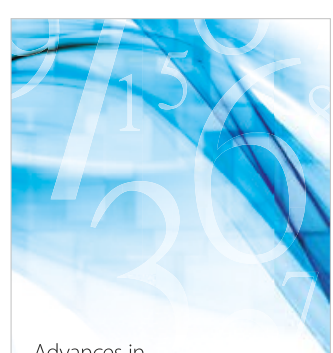

Advances in
Numerical Analysis
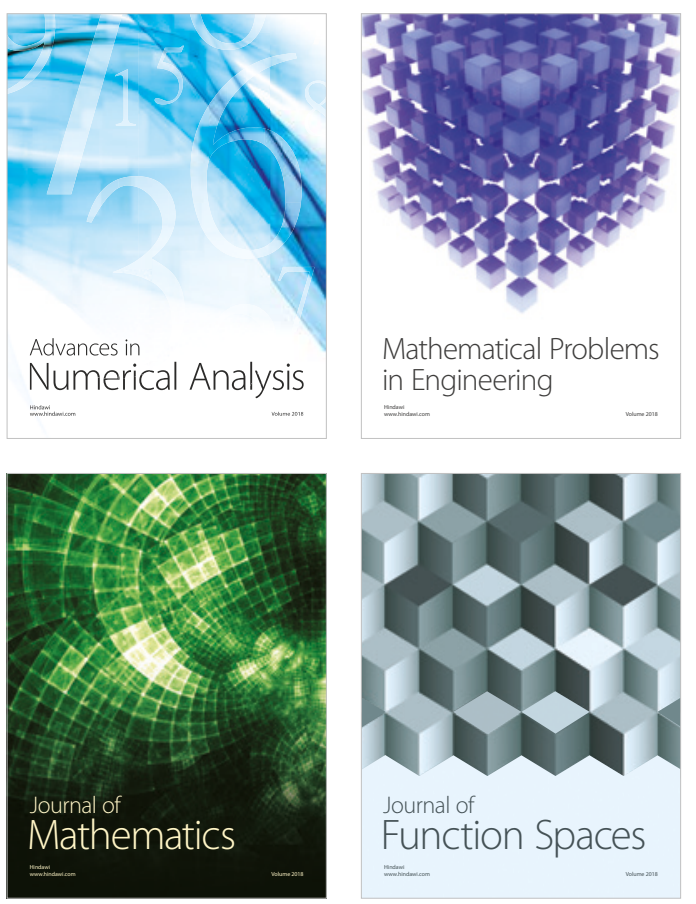

Mathematical Problems in Engineering

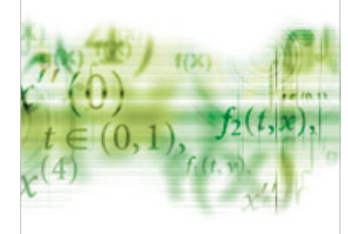

International Journal of

Differential Equations

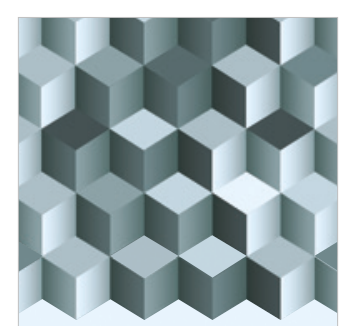

Journal of

Function Spaces
The Scientific

World Journal

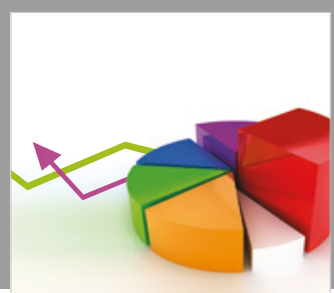

Journal of

Probability and Statistics
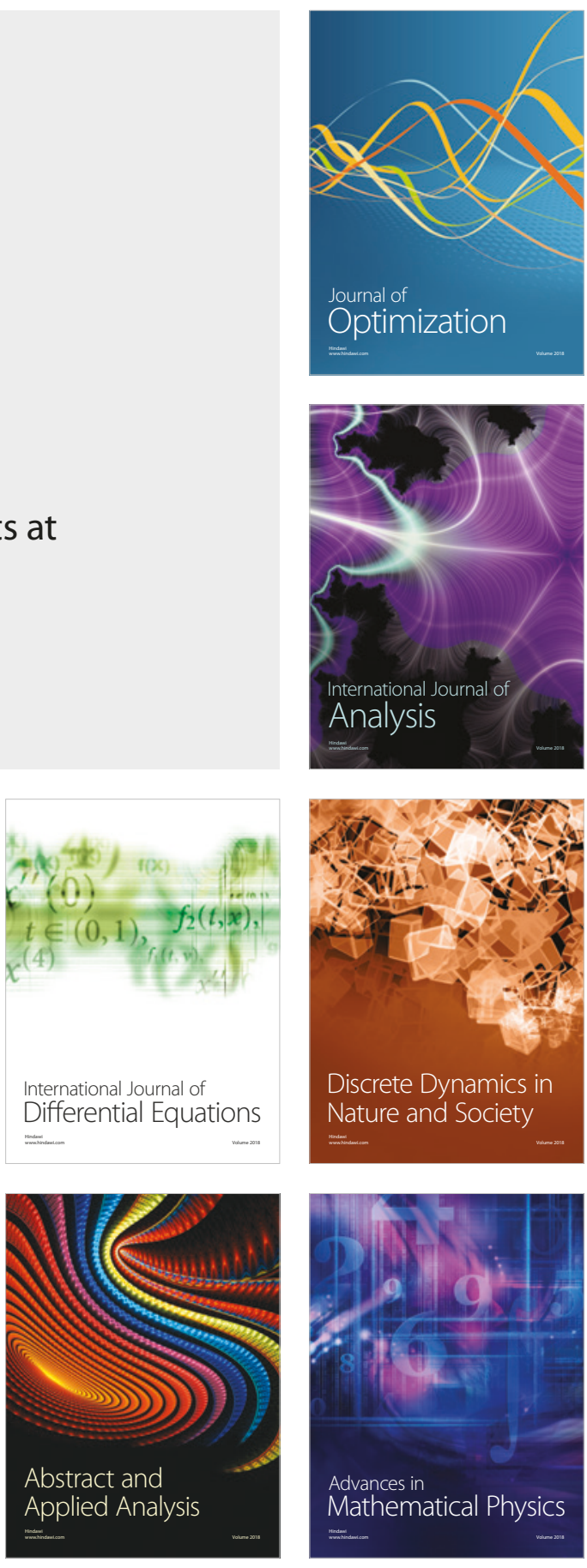